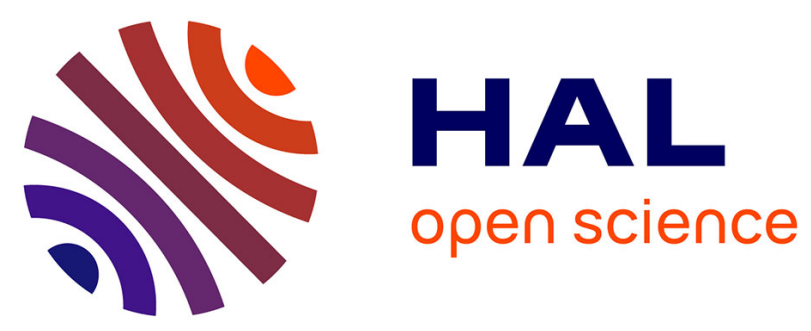

\title{
3-D Characterization of Urban Areas Using High-Resolution Polarimetric SAR Tomographic Techniques and a Minimal Number of Acquisitions
}

Yue Huang, Laurent Ferro-Famil

\section{- To cite this version:}

Yue Huang, Laurent Ferro-Famil. 3-D Characterization of Urban Areas Using High-Resolution Polarimetric SAR Tomographic Techniques and a Minimal Number of Acquisitions. IEEE Transactions on Geoscience and Remote Sensing, 2021, 59 (11), pp.9086-9103. 10.1109/TGRS.2020.3044150 . hal02297035

\section{HAL Id: hal-02297035 \\ https://hal.science/hal-02297035}

Submitted on 26 Jan 2021

HAL is a multi-disciplinary open access archive for the deposit and dissemination of scientific research documents, whether they are published or not. The documents may come from teaching and research institutions in France or abroad, or from public or private research centers.
L'archive ouverte pluridisciplinaire HAL, est destinée au dépôt et à la diffusion de documents scientifiques de niveau recherche, publiés ou non, émanant des établissements d'enseignement et de recherche français ou étrangers, des laboratoires publics ou privés. 


\title{
3-D Characterization of Urban Areas Using High-Resolution Polarimetric SAR Tomographic Techniques and a Minimal Number of Acquisitions
}

\author{
Yue Huang ${ }^{\circledR}$, Member, IEEE and Laurent Ferro-Famil ${ }^{\circledR}$, Member, IEEE
}

\begin{abstract}
This article addresses the 3-D reconstruction of urban areas using a minimal number of Synthetic Aperture Radar (SAR) acquisitions, that is, a set of three images, characterized by intermediate spatial resolution features. In such extreme conditions, conventional tomographic techniques reveal unadapted to refined 3-D imaging purposes, either due to the resulting intrinsic coarse vertical resolution, or to the low dimensionality of the data set, that prevents any separation of complex mixed scattering patterns. A new high-resolution (HR) tomographic estimator, based on a polarimetric signal subspace fitting criterion, is proposed to overcome these limitations, as this method adapts to the statistical behavior of the backscattered signals using robust metrics. The optimization of the corresponding focusing criterion is led through a new polarimetric alternating projection algorithm, characterized by a low computational cost, which may also be used to optimize the polarimetric the deterministic maximum likelihood criterion. The proposed polarimetric signal subspace fitting technique is shown to outperform the other studied HR techniques over both simulated signals and data acquired by the DLR's ESAR sensor at L-band over Dresden city, Germany. Finally full-rank polarimetric tomographic estimators are proposed that generalize nonparametric polarimetric estimators, and permit to estimate second-order polarimetric representations in 3-D, instead of unitary rank target vector with their conventional versions. This approach makes it possible to characterize polarimetric scattering mechanisms in 3-D.
\end{abstract}

Index Terms-Alternating projections, maximum likelihood (ML), Synthetic Aperture Radar (SAR) imaging, spectral analysis techniques, subspace fitting, urban remote sensing.

\section{INTRODUCTION}

$\mathbf{U}$ RBAN environments may be considered as 3-D media, whose response consists of a wide variety of coexisting and complex scattering mechanisms, such as double-bounce

Manuscript received January 2, 2020; revised May 6, 2020 and September 21, 2020; accepted December 1, 2020. This work was supported in part by the French National Research Agency (ANR), and in part by the Direction Générale de l'Armement (DGA) through the ALYS Project under Grant ANR-15-ASTR-0002. (Corresponding author: Yue Huang.)

Yue Huang is with the IETR Laboratory, University of Rennes 1, 35000 Rennes, France (e-mail: yue.huang@univ-rennes1.fr).

Laurent Ferro-Famil is with the IETR Laboratory, University of Rennes 1, 35000 Rennes, France and also with the CESBIO Laboratory, 31400 Toulouse, France.

Color versions of one or more figures in this article are available at https://doi.org/10.1109/TGRS.2020.3044150.

Digital Object Identifier 10.1109/TGRS.2020.3044150 scattering due to wall-ground reflections, surface scattering from roofs and from the ground, volumetric scattering over vegetated areas. Due to their intrinsic 2-D nature, Synthetic Aperture Radar (SAR) images cannot separate and characterize scattering contributions located at different elevations within a given range-azimuth resolution cell. SAR Interferometry (InSAR) may be used to estimate the topography of surfaces, but in the case of volumetric scenes, 2-D resolution cells may contain scatterers located at different elevations, and the characterization of such environments requires 3-D imaging capabilities. SAR tomography is a natural solution to the layover problem, and uses an additional aperture in elevation, in order to separate multiple scatterers within a 2-D resolution cell, from their specific phase patterns induced by spatial diversity. This 3-D imaging technique has been successfully applied to the characterization of man-made environments [1]-[7], using different data sets acquired by air- or spaceborne sensors, operated over various frequency bands.

As shown in [8], 3-D SAR focusing may be considered as a spectral estimation problem, which can be handled by numerous techniques. Classical Fourier-based methods represent the most basic kind of solution, and have been applied in [9] for the first demonstration of airborne SAR tomography. Due to practical factors, such as limited revisiting capabilities, acquisition costs, or temporal decorrelation, TomoSAR configurations usually consist of a moderate number of Multibaseline Interferometric SAR (MB-InSAR) images, measured with unevenly distributed baselines. In such cases, 3-D focusing using classical Fourier imaging techniques may lead to a poor vertical resolution, with vertical impulse responses having high sidelobes, and may fail to discriminate closely spaced scatterers. High-resolution (HR) spectral estimation methods, such as MUSIC, approaches based on Maximum Likelihood (ML) criteria, or other kinds of cost functions, can be used to resolve closely spaced scatterers in elevation, as shown in [10], [8], and [4]. These techniques are generally based on the analysis of the covariance matrix of the received MB-InSAR data, the estimation of which requires spatial multilooking, and leads to a gain of statistical robustness at the cost of a loss of horizontal resolution. Over spatially heterogeneous zones, such as dense urban areas, the accuracy of covariance matrix estimates may be strongly affected by 
the limited size of the sets of statistically stationary samples used to compute second-order statistics. Some neighborhood adaptive multidimensional SAR filtering methods [11], [12] have been designed to preserve point-like scatterers and fine textures, and reduce the speckle effect over complex environments. The impact of nonlocal adaptive filtering on 3-D reconstruction from TomoSAR data has been studied in [7] and [13]. The reconstruction of urban areas in 3-D using spaceborne SAR sensors recently experienced an important development, with the advent of HR SAR missions, such as TerraSAR-X (TSX) [3]. Spaceborne observation modes can produce time series of acquisitions resulting in large stacks of images, that may be used to estimate long-term ground motion [14], but also to build 3-D maps [1]-[3], over permanent or persistent scatterers whose response remains coherent over time. In this case, one may choose to resort to sparse signal analysis techniques, also referred to as Compressed Sensing (CS) approaches, which aim to estimate, under a set of conditions, the response of scatterers from a single snapshot, i.e., without spatial averaging. Such techniques rely on both the hypothesis of a number of scatterers per resolution cell that is much smaller than the number of acquisitions, and on the low dimensionality of a nominal MB-InSAR response, which may be represented with a small number of parameters. Specific signal processing techniques, often based on convex optimization, estimate jointly the number of sources and their parameters using particular criteria, whose sparsity promoting character may be tuned with a user-defined parameter [2], [3]. Several studies indicate that, over urban areas, time series of approximately 30 spaceborne SAR images shall be employed in order to reach satisfying results with classical sparse signal analysis techniques [2], [3]. Nevertheless, many areas in the world have been imaged in an interferometric configuration a few times only, and using intermediate resolution SAR sensors. This article addresses this kind of situation and performs SAR tomographic reconstruction in the extreme case of a minimal number, i.e., three, of polarimetric SAR images acquired with an intermediate spatial resolution, e.g., $2 \mathrm{~m} \times 3 \mathrm{~m}$. Within one resolution cell, the number of dominant scattering responses becomes comparable with the number of available acquisitions, which invalidates the use of sparse signal analysis techniques. This work proposes to perform 3-D reconstruction using covariance-based spectral estimation techniques, operated with polarimetric diversity. Indeed, as shown in [4], [6], [15]-[17], the application of conventional polarimetric tomographic estimators for the purpose of 3-D mapping over built-up areas in this extreme TomoSAR configuration led to significantly improved results with respect to single-polarization techniques. Using several polarization channels resulted in an undeniable increase of performance improvements for the characterization of urban areas, both in terms of discrimination of layover scattering contributions and determination of their dominant scattering features. In the general case, i.e., without any particular assumption on the source signal statistics, $M=3$ acquisitions, composed of $N_{P}$ polarimetric channels, may be used to reconstruct up to $M-1=2$ sources having arbitrary and potentially equal polarimetric responses and up to $N_{P} M-1$ sources having all different polarimetric patterns. The first, more generic, option is used in this article. A tomographic technique, characterized by its statistical adaptivity was proposed in [18] in order to cope with the complex nature of scattering patterns encountered in dense environments such as urban areas. The Polarimetric Noise Subspace Fitting (P-NSF) estimator, inspired from the study [19], was proposed in [18], together with its analytical solution for the estimation of 3-D polarimetric parameters. Despite its rather good performance for SAR tomography purposes reported in [18], this technique, due to its use of noise subspace of the observed signal, meets limitations in the presence of highly coherent scatterers. To overcome this limitation, a new model-adaptive estimator, named Polarimetric Signal Subspace Fitting (P-SSF), is proposed in this article. As has been reported in the past studies, parameter estimation using polarimetric multidimensional tomographic estimators has to face the issue of computational complexity, which was maintained at a low level in the case of the P-NSF in [18], [19]. Therefore, a new alternating maximization algorithm, named Polarimetric Alternating Projection (P-AP), is proposed to solve the optimization of the P-SSF focusing criterion, which can be implemented as an iterative mono-dimensional approach with computational attractiveness. It is noteworthy that the proposed P-AP algorithm can be used as a solution for the Polarimetric Deterministic Maximum Likelihood (P-DML) technique too. Similar to conventional ones, described in [4], [15], [18], the proposed polarimetric approaches, P-SSF and P-DML, estimate, for each detected and localized scatterer, a polarimetric target vector, i.e., a unitary-rank polarimetric representation. This information can be effectively used to characterize scattering environments with a low polarimetric randomness. As this was illustrated in [20], intermediate resolution polarimetric SAR images measured over urban environments reveal very complex features, due to the mixing of very different scattering mechanisms within a single resolution cell. In such a case, unitary-rank representations are not well adapted to handle the whole polarimetric information and second-order polarimetric statistics have to be estimated, in order to provide a full-rank (FR) polarimetric representation. FR polarimetric tomographic techniques have been proposed in [21], and used for 3-D imaging of underfoliage targets in [22], [23], or over forested areas in [24]. A thorough derivation of FR polarimetric and nonparametric estimators is provided, which allows one to characterize the properties of 3-D polarimetric second-order representations using classical tools. This article is organized as follows. In Section II, tomographic signal models and focusing techniques are presented for a minimal TomoSAR configuration in both scalar and polarimetric cases. The performance of commonly used tomographic estimation techniques is illustrated using numerical simulations. A case study based on a dual-baseline PolInSAR data set is investigated to demonstrate the need for HR adaptive polarimetric estimators and FR polarimetric analysis in practice. The polarimetric SSF and DML estimators are derived in Section III and their performance is evaluated using simulations and L-band data acquired by the DLR's E-SAR sensor over the test site of Dresden. Section IV describes FR PolTomoSAR techniques and the

\section{ACCEPTED MANUSCRIPT}


TABLE I

List of ACRONYMs of Methods And Models

$\begin{array}{ll}\text { BF } & \text { Beamforming } \\ \text { CP } & \text { Capon's method } \\ \text { MUSIC } & \text { MUltiple SIgnal Classification } \\ \text { WSF } & \text { Weighted Subspace Fitting } \\ \text { SSF } & \text { Weighted Signal Subspace Fitting } \\ \text { NSF } & \text { Weighted Noise Subspace Fitting } \\ \text { DML } & \text { Deterministic Maximum Likelihood } \\ \text { SML } & \text { Stochastic Maximum Likelihood } \\ \text { UM } & \text { Unconditional signal Model } \\ \text { CM } & \text { Conditional signal Model } \\ \text { AP } & \text { Alternating Projection } \\ \text { FR } & \text { Full Rank } \\ \text { P-X } & \text { Polarimetric version of method X }\end{array}$

application of FR polarimetric Capon's approach to the test data set. In particular, it is shown that this approach is able to separate entangled 3-D features of vegetated areas and builtup structures in dense urban areas. The acronyms of methods and models used in this article are listed in Table I.

\section{Assessment of SAR TOMOgRaphy Performed USING A Minimal CONFIGURATION}

This section aims to assess the performance of existing SAR tomographic focusing techniques in the particular case of a minimal configuration, i.e., using a stack composed of three images. Comparisons are led in order to evaluate the ability of these techniques to discriminate and characterize objects with respect to various factors such as vertical resolution, statistical properties of SAR echoes, or polarimetric scattering patterns. The results of this analysis are then used to define the features a performing estimator should possess in the specific case under study. The derivation of such an estimator is the object of the section after the present one.

\section{A. Tomographic Signal Models}

1) Single-Polarization TomoSAR Signal Models: Considering an azimuth-range resolution cell that contains $n_{s}$ scatterers located at different heights, the data vector measured over $M$ SAR acquisitions, $\mathbf{y} \in \mathbb{C}^{M \times 1}$, can be formulated as the sum of the backscattered contributions, as follows:

$$
\mathbf{y}(l)=\sum_{i=1}^{n_{s}} s_{i}(l) \mathbf{a}\left(z_{i}\right)+\mathbf{n}(l)=\mathbf{A}(\mathbf{z}) \mathbf{s}(l)+\mathbf{n}(l)
$$

where $l=1, \ldots, L$ indicates one of the $L$ independent realizations of the signal acquisition, also called looks. The source signal vector, $\mathbf{s}(l)=\left[s_{1}(l), \ldots, s_{n_{s}}(l)\right]^{T}$, contains the complex reflection coefficient of each scatterer, related to its reflectivity through $p_{i}=\mathrm{E}\left(\left|s_{i}(l)\right|^{2}\right)$, whereas the interferometric phase information associated with a source located at the elevation position $z$ above the reference focusing plane, is given by the steering vector

$$
\mathbf{a}(z)=\left[1, \exp \left(j k_{z_{2}} z\right), \ldots, \exp \left(j k_{z_{M}} z\right)\right]^{T}
$$

where $k_{z_{m}}=(4 \pi / \lambda)\left(B_{\perp_{m}} / r_{1} \sin \theta\right)$ is the two-way vertical wavenumber between the master and the $m$ th acquisition tracks. The carrier wavelength is represented by $\lambda$ and $B_{\perp_{m}}$ is the perpendicular baseline, whereas $\theta$ stands for the incidence angle and $r_{1}$ is the slant range distance between the master track and the scatterer. The steering matrix gathers the interferometric phases of all the sources, and is written as $\mathbf{A}(\mathbf{z})=\left[\mathbf{a}\left(z_{1}\right), \ldots, \mathbf{a}\left(z_{n_{s}}\right)\right]$, with $\mathbf{z}$ the vector of unknown source heights. The complex additive noise, $\mathbf{n} \in \mathbb{C}^{M \times 1}$ is assumed to follow a Gaussian distribution and to be white in time and space, i.e., $\mathbf{n} \sim \mathcal{N}\left(\mathbf{0}, \sigma_{n}^{2} \mathbf{I}_{(M \times M)}\right)$ and $\mathrm{E}\left(\mathbf{n}(l) \mathbf{n}^{H}(k)\right)=$ $\sigma_{n}^{2} \mathbf{I}_{(M \times M)} \delta_{l, k}$. The covariance matrix of the received signal (1) is generally expressed as $\mathbf{R}=\mathrm{E}\left(\mathbf{y} \mathbf{y}^{H}\right)=\mathbf{A} \mathbf{R}_{\mathrm{ss}} \mathbf{A}^{H}+\sigma_{n}^{2} \mathbf{I}_{M \times M}$, where the source covariance matrix is $\mathbf{R}_{\mathrm{ss}}=\mathrm{E}\left(\mathbf{s s}^{H}\right)$, and $\mathbf{p}=\operatorname{diag}\left(\mathbf{R}_{\mathrm{ss}}\right)=\left[p_{1}, \ldots, p_{n_{s}}\right]^{T}$.

Depending on the nature of the observed medium, the composite signal $\mathbf{y}(l)$ may follow different behaviors linked to the statistical properties of the source signal $\mathbf{s}(l)$, as described in [18]. For distributed scatterers, the observed signal behavior may be represented by the Unconditional Model (UM) [25], which accounts for the random nature of the source signal, using a multiplicative Gaussian noise term. This type of behavior is suitable to model speckle-affected responses over distributed environments, such as rough surfaces, ground, and natural volumes. Deterministic scatterers have a highly coherent scattering response, which may be represented using the Conditional Model (CM) [25], which assumes a frozen source signal over all the observations. This behavior is generally related to specular or well-localized scattering mechanisms and can be observed over coherent scatterers like calibrators, facets facing the radar, double-bounce reflections over dihedral-like objects having smooth surfaces or may be linked to resonant behaviors over quasi-periodic media [20], [26]. Under both UM and CM assumptions, the received signal $\mathbf{y}(l)$ follows a complex multivariate gaussian distribution, but with different likelihood functions

2) PolTomoSAR Signal Models: The polarimetric response of a scatterer may be represented by an $N_{P}$-element data vector, $\mathbf{v}$, containing the complex scattering coefficients measured for different transmitted and received polarization states. In a monostatic configuration, and using the well-known Pauli polarization basis [27], this vector can be written as $\mathbf{v}=$ $\frac{1}{\sqrt{2}}\left[S_{\mathrm{hh}}+S_{\mathrm{vv}}, S_{\mathrm{hh}}-S_{\mathrm{vv}}, 2 S_{\mathrm{hv}}\right]^{T}$, where $S_{\mathrm{xy}}$, with x and y equal to $\mathrm{h}$ or $\mathrm{v}$, represents one element of the $(2 \times 2)$ scattering matrix $\mathbf{S}$. Depending on the polarimetric configuration at hand, the number of available channels verify $1 \leq N_{P} \leq 4$. Over complex urban environments, both interferometric and polarimetric scattering fluctuations of speckle-affected responses can be taken into account by using $\mathbf{v}(l)=s(l) \mathbf{k}(l)$, where $p=\mathrm{E}\left(\mathbf{v}(l)^{H} \mathbf{v}(l)\right)$ represents the span [27], or polarimetric reflectivity, of the scatterer's response, and $\mathbf{k}(l) \in \mathbb{C}^{N_{P} \times 1}$ is a target vector containing a normalized polarimetric information, $\mathbf{k}^{H}(l) \mathbf{k}(l)=1$. Classical incoherent Polarimetric SAR (PolSAR) analysis is usually derived from the secondorder statistics of polarimetric data, i.e., from the covariance matrix of the polarimetric responses

$$
\mathbf{T}=\mathrm{E}\left(|s|^{2} \mathbf{k k}^{H}\right)
$$

with $1 \leq \operatorname{rank}(\mathbf{T}) \leq N_{P}$, depending on the observed medium.

In the unitary-rank case, the relative information between the polarimetric channels is deterministic, and the polarimetric 
response writes $\mathbf{v}(l)=s(l) \mathbf{k}$. Signals measured in a Multibaseline PolInSAR configuration are then gathered in an $N_{P} M$-element Polarimetric Tomographic received signal, $\mathbf{y}_{P}$, is formed by stacking the TomoSAR responses for each polarization channel, as $\mathbf{y}_{P}=\left[\mathbf{y}_{1}^{T}, \ldots, \mathbf{y}_{N_{P}}^{T}\right]^{T} \in \mathbb{C}^{N_{P} M \times 1}$, where $\mathbf{y}_{q} \in \mathbb{C}^{M \times 1}$ represents the TomoSAR response for the $q$ th polarimetric channel, i.e., $\left[\mathbf{y}_{q}\right]_{j}=\left[\mathbf{v}_{j}\right]_{q}\left(q=1, \ldots, N_{P}\right)$. Using this convention, the polarimetric steering vector of the $i$ th source, i.e., the ideal interferometric response with unitary polarimetric target vector $\mathbf{k}_{i}$, is given by

$$
\mathbf{a}\left(z_{i}, \mathbf{k}_{i}\right)=\left(\mathbf{I}_{N_{P} \times N_{P}} \otimes \mathbf{a}\left(z_{i}\right)\right) \mathbf{k}_{i}=\mathbf{B}_{\mathbf{a}}\left(z_{i}\right) \mathbf{k}_{i}
$$

where $\otimes$ stands for the Kronecker product operator. Similar to the single polarization case, the received PolTomoSAR signal $\mathbf{y}_{P}(l)$ may be formulated as

$$
\begin{aligned}
\mathbf{y}_{P}(l) & =\sum_{i=1}^{n_{s}} s_{i}(l) \mathbf{a}\left(z_{i}, \mathbf{k}_{i}\right)+\mathbf{n}(l) \\
& =\mathbf{A}(\mathbf{z}, \mathbf{K}) \mathbf{s}(l)+\mathbf{n}(l) \in \mathbb{C}^{N_{P} M \times 1}
\end{aligned}
$$

with $\mathbf{A}(\mathbf{z}, \mathbf{K})=\left[\mathbf{a}\left(z_{1}, \mathbf{k}_{1}\right), \ldots, \mathbf{a}\left(z_{n_{s}}, \mathbf{k}_{n_{s}}\right)\right] \in \mathbb{C}^{N_{P} M \times n_{s}}$ the polarimetric steering matrix. The UM and CM model assumptions described for single-polarization signals can be used in the PolTomoSAR case too, as shown in [18].

The FR PolTomoSAR signal model and the corresponding focusing techniques are described in detail in Section IV.

\section{B. Single Polarization Tomography}

1) Common Single Polarization Tomographic Techniques: The objective of single polarization tomographic focusing concerns the estimation of $\mathbf{z}$ and $\mathbf{p}$, the elevation and reflectivity of the $n_{s}$ measured scatterers, from $L$ realizations of the MB-InSAR signal, $\{\mathbf{y}(l)\}_{l=1}^{L}$. This inverse problem may be solved using spectral analysis techniques [6], [8], [17], [18], [28], based on the $L$-look estimate of the data covariance matrix, $\widehat{\mathbf{R}}=(1 / L) \sum_{l=1}^{L} \mathbf{y}(l) \mathbf{y}^{H}(l)$. The wide variety of existing spectral analysis methods may be categorized according to some of their features, such as their processing principle, performance, and complexity [29]-[31].

a) Mono-dimensional approaches: They determine $\widehat{\mathbf{z}}$ as the coordinates of the local maxima of a continuous monodimensional objective function $P(z)$, whose generic form is given by

$$
P(z)=\left(\mathbf{a}^{H}(z) \mathbf{Q}^{p} \mathbf{a}(z)\right)^{q}
$$

and hence have a low computational complexity, which does not depend on the number of scatterers $n_{s}$. Within this category, nonparametric techniques, such as the Fourier Beamformer (BF), with $\mathbf{Q}=\widehat{\mathbf{R}}, p=q=1$ in (6), and Capon's method (CP) [32], with $\mathbf{Q}=\widehat{\mathbf{R}}, p=q=-1$ in (6), are commonly applied for tomographic focusing [8], [10], [33], [34]. Both $\mathrm{BF}$ and $\mathrm{CP}$ are known to be relatively robust to focusing artifacts, generally implied by problematic acquisition conditions or scattering behaviors, but have a coarse resolution, limited by the range spanned by the $\left\{k_{z_{i}}\right\}_{i=1}^{M}$ values at hand. With respect to $\mathrm{BF}, \mathrm{CP}$ possesses, for high enough Signal-to-Noise Ratio (SNR), improved resolution, and sidelobe levels, at the cost of a reduced radiometric accuracy. MUSIC [35] is a parametric HR technique [36], characterized by a much improved resolution, which does not directly depend on SNR or baseline distribution, but rather on the quality of the estimation of $\mathbf{R}$. The mono-dimensional objective function is given, in the case of MUSIC, by (6) with $\mathbf{Q}=\widehat{\mathbf{E}}_{n} \widehat{\mathbf{E}}_{n}^{H}, p=1, q=-1, \widehat{\mathbf{E}}_{n}$ being a matrix formed by the eigenvectors of $\widehat{\mathbf{R}}$ corresponding to its $M-\hat{n}_{s}$ smallest eigenvalues. Being based on subspace orthogonality, MUSIC is known to perform badly in the presence of correlated scatterer responses [29], and, as a parametric approach, it requires to estimate the model order $\hat{n}_{s}$, whereas model order selection may be performed a posteriori for $\mathrm{BF}$ and $\mathrm{CP}$. The reflectivity vector, $\mathbf{p}$, may be estimated directly from $P(z)$ for $\mathrm{BF}$ and $\mathrm{CP}$, whereas an additional processing step, typically a Least-Square (LS) estimation [36], [37], is required for MUSIC.

b) Multidimensional techniques: They aim to jointly estimate the features of the $n_{s}$ scatterers, the number to be estimated, through the optimization of a concentrated criterion, $Q(\mathbf{z}), \mathbf{p}$ being generally estimated implicitly [25], [38]. Such parametric techniques are more robust and accurate than mono-dimensional estimators, as they account for the complex interactions between the different responses, but at an expensive computational cost, which increases with $\hat{n}_{s} \leq M-1$, the search dimension. Most of the multidimensional HR techniques able to cope with irregular $k_{z_{i}}$ distribution, are inspired by the ML approach, which determines the parameters maximizing a likelihood function, established under the Unconditional (UM) or CM assumption [38]. The resulting Stochastic (SML) or Deterministic ML (DML) estimators are characterized by a very high resolution, the DML estimator being statistically less efficient than SML, especially for highly correlated source signals [25]. ML techniques show, in most cases, better performance than MUSIC, with a higher computational cost [39]. One may note that the approach proposed in [40] is not utilized in this study, as it optimizes an SML criterion under a very strong simplifying the assumption, considering that all the scatterers to be discriminated within a range resolution cell have exactly the same complex reflection coefficient, $s_{i}$ in (1). Using the SML criterion, and the underlying UM statistical behavior, the assumed property of equal reflectivities is verified with probability 0 , and hence may generate distortions. Weighted Subspace Fitting (WSF) techniques were introduced in [29], [30], [38], and [41] in order to overcome limitations of ML approaches in terms of computational complexity, and, more importantly, of performance when dealing with a finite number of looks, i.e., when using $\widehat{\mathbf{R}}$ instead of $\mathbf{R}$, or assuming an erroneous model order, $\hat{n}_{s}$. They estimate source parameters based on the LS fitting between modeled and estimated noise or signal subspaces, $\widehat{\mathbf{E}}_{n}$ or $\widehat{\mathbf{E}}_{s}$, respectively. The use of a weighting term allows one to compensate discrepancies between true and estimated subspace features, leading to the NSF or SSF multidimensional criteria, $Q_{N S F}(\mathbf{z})=\left\|\widehat{\mathbf{E}}_{n}^{H} \mathbf{A}(\mathbf{z})\right\|_{\mathbf{W}}^{2}$ and $Q_{S S F}(\mathbf{z})=\| \widehat{\mathbf{E}}_{s}-$ $\mathbf{A}(\mathbf{z}) \mathbf{T} \|_{\mathbf{W}}^{2}$ where $\|\mathbf{x}\|_{\mathbf{W}}^{2} \triangleq \mathbf{x}^{H} \mathbf{W} \mathbf{x}$. For specific values of the weighting matrix, $\mathbf{W}$, the NSF or SSF techniques can be asymptotically associated with other spectral estimators, such

\section{ACCEPTED MANUSCRIPT}


as a multidimensional version of MUSIC, or ML methods [29]. In particular, a consistent $\mathbf{W}$ value permits one to obtain minimum variance estimates, which asymptotically reach the Cramér-Rao Lower Bound (CRLB) [38], and hence adapt to the statistics of the measured sources [6], [18], [25]. WSF approaches are asymptotically equivalent to the SML technique, but with a reduced computation cost [38]. In the case of low SNR conditions, the SSF technique provides better estimation accuracy than the SML [25]. For a specific value of $\mathbf{W}$, the SSF estimator is asymptotically equivalent to, and outperforms, the DML technique [41]. One may note that similar to the MUSIC approach, the performance of the NSF estimator may be affected by model order mismatch [38]. As this is shown in Section II-B2, ML and WSF approaches allow to reach very good performance in extreme conditions, but the numerical evaluation of their objective functions may require some particular attention and specific steps, such as intermediate decompositions [38], in order to avoid divergent behaviors occasioned by avoidable numerical instabilities, and not to the approaches themselves.

2) Performance Assessment: The performances of the different aforementioned tomographic estimators are compared in the particular case of a small number of acquisitions, $M$. MB-InSAR signals are simulated for two scatterers, separated by a lag in elevation denoted $\Delta z$, and whose source terms verify $p_{i}=1$, and $\left|\mathrm{E}\left(s_{1} s_{2}^{*}\right)\right|=\rho$. Acquisition noise is considered, with SNR, SNR $=\frac{p_{i}}{\sigma_{n}^{2}}$ equal to $20 \mathrm{~dB}$ as a default value. Simulations are realized with 500 independent trials. A wide variety of signal models may then be simply simulated by setting $\rho=0.995$ for coherent (CM) signals, $\rho=0$ for uncorrelated ones (UM), intermediate values for hybrid behaviors [6], [17], [18]. The geometry of the $M$ SAR acquisitions is characterized by a regular distribution of $k_{z_{i}}$, with spacing $\Delta k_{z}=0.2$, leading to a vertical resolution of $\delta_{z}=10 \mathrm{~m}$ for $M=3$ and $\delta_{z}=5 \mathrm{~m}$ for $M=6$. Due to its poor resolution and its inability to separate signals in the investigated configurations, the BF technique is not considered in this evaluation.

a) Asymptotic properties: Simulations are run with two UM scatterers, with $\rho=0$ and $\Delta z=4 \mathrm{~m}$, and the data covariance matrix is estimated with a different number of looks $L$, in order to investigate the asymptotic properties of the investigated techniques. Fig. 1 illustrates the performances in terms of Root Mean Squared Error (RMSE) in elevation, as a function of $L$. CP shows the worst performance, due to a limited vertical resolution, whereas HR estimators, such as MUSIC and WSF techniques, are asymptotically equivalent to $\mathrm{ML}$ techniques for large sample size values, i.e., $L=256$ here. Using an intermediate-resolution TomoSAR configuration, i.e., $M=6$ with $\delta_{z}=5 \mathrm{~m}$, these approaches achieve extremely good performance even with a low number of looks. For a minimal TomoSAR configuration, i.e., $M=3$ with $\delta_{z}=10 \mathrm{~m}$, a deterioration of the performance is observed, and HR methods provide a significant improvement compared to Capon's technique. One may note in Fig. 1 that in both cases, $L=256$ appears as a number of looks for which all techniques have converged to their asymptotic behavior,

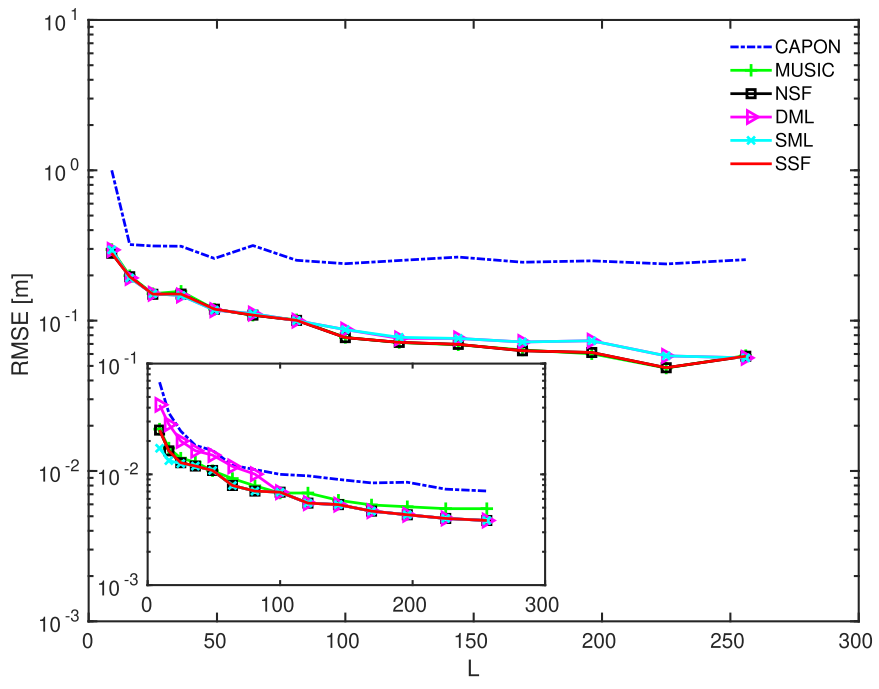

Fig. 1. Height RMSE for a varying number of looks $L . \rho=0, \Delta z=4 \mathrm{~m}$. (Outer Plot) $\delta_{z}=10 \mathrm{~m}(M=3)$. (Inner Plot) $\delta_{z}=5 \mathrm{~m}(M=6)$.

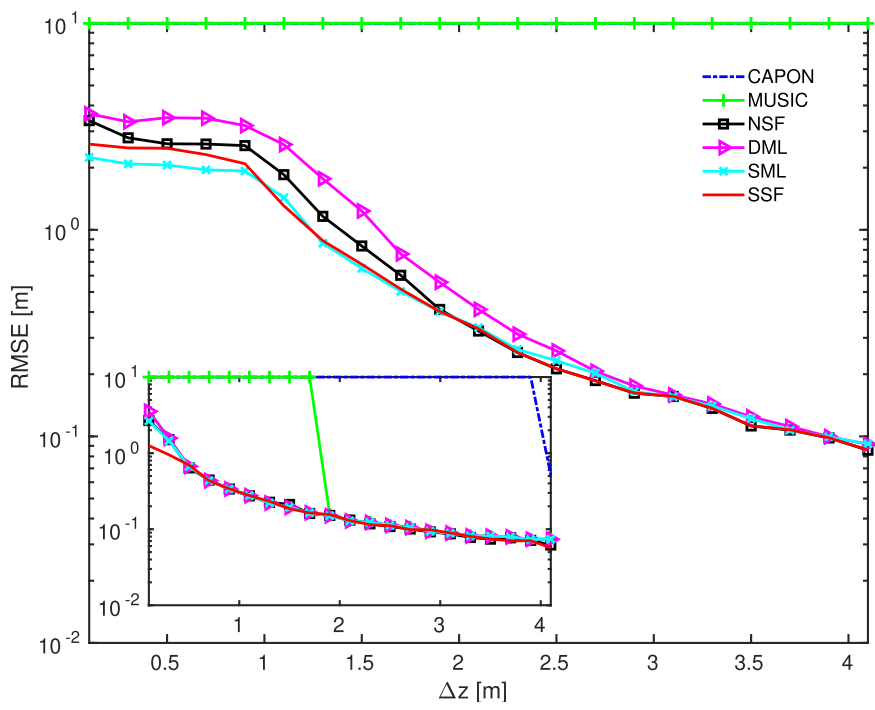

Fig. 2. Height RMSE of $\hat{z}$ in a minimal configuration for varying source separation $\Delta z$ with $M=3\left(\delta_{z}=10 \mathrm{~m}\right)$. (Outer Plot) $\rho=0.995$. (Inner Plot) $\rho=0$.

and this number of looks is selected as the default value in the following sections. Compared to other HR estimators, the performance of the DML technique degrades significantly, as $L$ reaches insufficient values, whereas WSF techniques lead to good estimates whatever the number of looks.

b) Estimation accuracy and source correlation: Fig. 2 shows the performance of the studied estimators in the lowresolution case, $M=3\left(\delta_{z}=10 \mathrm{~m}\right)$, for different source correlations $\rho$ and separations $\Delta z$. For uncorrelated scatterers, $\rho=0$, the results plotted in Fig. 2(a) indicate that, as a mono-dimensional method, the MUSIC estimator fails in the presence of closely spaced scatterers, with $\Delta z<2 \mathrm{~m}$, whereas multidimensional methods, like WSF and ML techniques, show a more robust behavior. In the presence of coherent scatterers, Fig. 2(b) depicts a degraded performance for all

\section{ACCEPTED MANUSCRIPT}




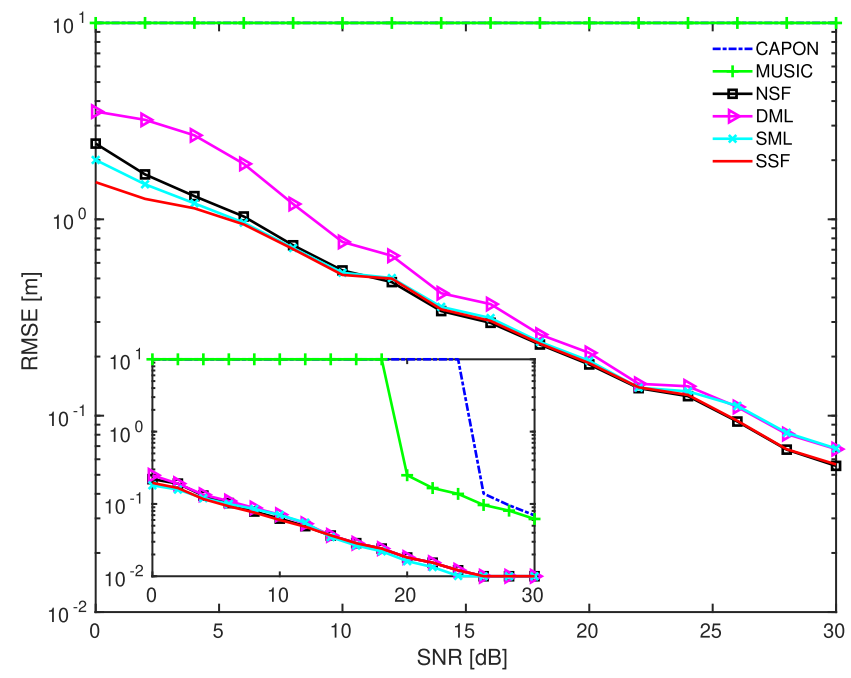

Fig. 3. Height RMSE for varying SNR values and different array size. $\rho=0.995, \Delta z=4 \mathrm{~m}$. (Outer Plot) $M=3$. (Inner Plot) $M=6$.

methods. Capon and MUSIC techniques fail completely, since the source signal covariance matrix, $\widehat{\mathbf{R}}_{s s}$, tends to be singular. The NSF estimator performs better than the DML technique, whereas the SSF estimator outperforms all the other techniques, whatever the correlation, due to its model-adaptive nature conferred by the fitting matrix $\mathbf{W}$ used in the WSF criterion.

c) Coherent scatterers and SNR: Simulations are run with CM scatterers and for varying SNR values. One may observe in Fig. 3 that much better results are obtained in the intermediate-resolution case, $M=6\left(\delta_{z}=5 \mathrm{~m}\right)$, compared to those of the low-resolution configuration, $M=3\left(\delta_{z}=10 \mathrm{~m}\right)$. Divergences between the characteristics of the HR techniques are observed when the resolution and SNR values are low. Fig. 3 shows that, using a minimal TomoSAR configuration, Capon and MUSIC estimators have a significantly degraded performance. The NSF technique performs similar to SML, and better than the DML estimator, when SNR $<20 \mathrm{~dB}$. The SSF estimator outperforms the other techniques, whatever the SNR value.

\section{Polarimetric Tomography}

Compared to its single-polarization counterpart, Polarimetric SAR Tomography (PolTomoSAR) is expected to improve the performance of 3-D focusing. In particular, polarization diversity helps to further discriminate scattering sources, and may reveal particularly useful in the case of low vertical resolution and configurations with a small number of images. Moreover, such an imaging mode offers the possibility to map and identify scattering behaviors in 3-D. However, limitations regarding the handling of potentially complex stochastic scattering using spectral analysis techniques such as those mentioned in the SP case, are still relevant for polarimetric configurations.

1) Common Polarimetric Tomographic Techniques: Polarimetric SAR tomography aims to estimate not only the elevation $z_{i}$ and reflectivity $p_{i}$ of each source, but also its scattering vector $\mathbf{k}_{i}$, a unitary $N_{P}$-element complex vector describing how the considered source reflects impinging waves for each polarimetric channel. Several multivariate spectral analysis techniques, based on the data $\left(N_{P} M \times N_{P} M\right)$ covariance matrix, $\widehat{\mathbf{R}}_{P}$, can be found in the literature [4], [19], [42], [43], and may be qualified as unitary-rank polarimetric tomographic estimators, since the retrieved polarimetric information consists of a target vector, as opposed to FR techniques, estimating polarimetric covariance matrices, and presented later in this article. Mono-dimensional Polarimetric (P-) techniques, such as P-BF, P-CP, and P-MUSIC, use a 1-D objective function $P(z, \mathbf{k})=\left(\mathbf{a}^{H}(z, \mathbf{k}) \mathbf{Q}^{p} \mathbf{a}(z, \mathbf{k})\right)^{q}$, whose polarimetric optimization, for each $z$ value, generally relies on the eigendecomposition of a $\left(N_{P} \times N_{P}\right)$ matrix, in order to determine the target vector $\mathbf{k}(z)$. Compared to the SP case, the computational complexity of such P-tomographic techniques is only multiplied by a small factor, and remains particularly attractive.

Multidimensional approaches, such as the P-ML and P-WSF estimators, may be formulated from their single polarization expressions by replacing the steering matrix $\mathbf{A}(\mathbf{z})$ with $\mathbf{A}(\mathbf{z}, \mathbf{K})$. The height and polarimetric parameters, $\mathbf{z}, \mathbf{K}$, may be estimated by optimizing the cost function $Q(\mathbf{z}, \mathbf{K})$ over a $\left(2 N_{P}-1\right) \hat{n}_{s}$-dimensional space, which implies an excessive computational burden. There exist computationally efficient solutions for solving ML criteria techniques in single polarization case [38], [44], but they have not been extended to the polarimetric case. Two versions of the P-ML technique proposed in [42] and [15] rely on the definition of an approximate cost function with a scalar argument and hence suffer from the drawbacks of mono-dimensional approaches. Regarding WSF techniques, an analytical solution for the polarimetric NSF estimator has been proposed in [19] and [18], which permits to maintain the computational cost close to the one of the single polarization case, and relies on an $\hat{n}_{s}$-dimensional search. As has been shown in the scalar case, the SSF estimator outperforms other techniques due to its adaptivity to complex scattering and statistical features. It is thus expected that the extension of the SSF technique to the polarimetric case may be of great interest for 3-D focusing purposes and a computationally efficient solution is proposed in this article.

2) Performance Assessment: Numerical illustrations given in this section are based on the baseline configuration used in the single polarization case, and deals with fully Polarimetric (P) acquisitions, $N_{P}=3$.

a) Use of polarimetric diversity: This case study considers two scatterers, with uncorrelated responses with equal polarimetric covariance matrices, $\mathbf{T}_{1}=\mathbf{T}_{2}=\mathbf{T}$, characterized by a degree of polarimetric scattering randomness, $H$, varying from 0 to 1 . The polarimetric entropy is defined in [45] as $H(\mathbf{T})=-\sum_{q=1}^{N_{P}} \tilde{\lambda}_{q} \log _{N_{P}} \tilde{\lambda}_{q}$ where $\tilde{\lambda}_{q}=\lambda_{q} / \sum_{i=1}^{N_{P}} \lambda_{i}$, with $\lambda_{q}$ an eigenvalue of the polarimetric covariance matrix T defined in (3). For a deterministic polarimetric responses, i.e., $\mathbf{k}(l)=\mathbf{k}_{0}, \forall l$ in (3), the polarimetric covariance matrix has a unitary rank and $H=0$. Completely unpolarized responses, i.e., polarimetric white noise, are characterized by a white covariance matrix, i.e., $\lambda_{q}=\lambda_{1}, \forall q$, and $H=1$. In a general case, $0 \leq H \leq 1$. The relevance of polarimetric data processing diversity is evaluated by considering a system working with a single polarization channel, represented by an $N_{P}$-element complex vector $\mathbf{k}_{a}$ that takes into

\section{ACCEPTED MANUSCRIPT}




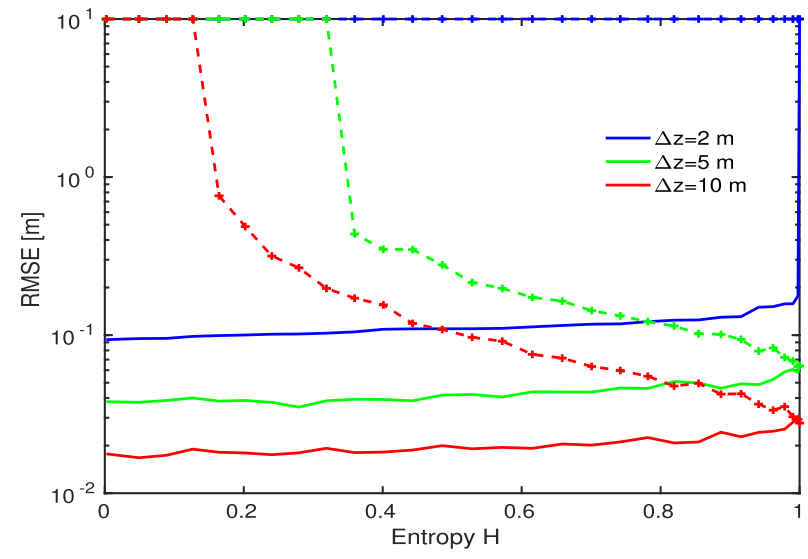

Fig. 4. Height RMSE of the MUSIC estimator with two sources in the most favorable (solid) and least favorable (+ dash) polarization selection cases. $M=3\left(\delta_{z}=10 \mathrm{~m}\right), \rho=0$.

account specific antenna polarization states at both transmit and receive. Hence, for a specific polarimetric configuration of the system, the received signal may be formulated as a linear transformation of its polarimetric counterpart as $\mathbf{y}=$ $\left(\mathbf{k}_{a} \otimes \mathbf{I}_{M \times M}\right)^{H} \mathbf{y}_{P}$. Two characteristic polarimetric configurations may be considered, i) in the most favorable case, the acquisition polarization state is parallel to the eigenvector of $\mathbf{T}, \mathbf{k}_{\max }$, corresponding to the largest eigenvalue, i.e., $\left|\mathbf{k}_{a}^{H} \mathbf{k}_{\max }\right| /\left(\left\|\mathbf{k}_{a}\right\|\left\|\mathbf{k}_{\max }\right\|\right)=1$ and the SNR on the receiver is maximal; ii) in the least favorable case, these target vectors are orthogonal, i.e., $\mathbf{k}_{a}^{H} \mathbf{k}_{\max }=0$, and the SNR is minimal. Fig. 4 shows that using MUSIC in the most favorable case leads to performances that do not depend significantly on the entropy value, meaning that optimally tuning the polarimetric channel to $\mathbf{k}_{a} \propto \mathbf{k}_{\max }$ is the best solution, in general. Using a fixed polarization, or, at least, a nonoptimal one, may lead to a severe deterioration in performance, depending on the entropy of the observed polarimetric response. Indeed, the receiver's polarization state $\mathbf{k}_{a}$ may fall into, or close to, the null space of well polarized responses, with $H=0$, whereas when $H=1$, the energy of the backscattered response is equally distributed over polarimetric directions, and using polarimetric diversity does not permit to improve the SNR. Simple conclusions may be drawn from the curves plotted in Fig. 4. Using fully polarimetric acquisitions and polarimetrically adaptive processing permits to reach the performance indicated by the continuous plots, whereas working over a particular polarimetric channel leads to the performance lying between the continuous and dashed curves. One may note that polarimetric diversity is particularly useful for low $H$ values, typically reached over artificial objects, and when observing closely spaced scatterers.

b) Influence of the polarimetric distance: The sensitivity to polarimetric diversity of several spectral estimators used from tomographic focusing has been investigated in [18]. Following a similar evaluation protocol, one finds that the use of polarimetric diversity plays a major role, in terms of scatters' separation and height estimation accuracy, in extreme configurations, i.e., with a coarse resolution and for correlated responses. In such problematic situations, the subspace fitting technique, P-NSF, outperforms the other HR techniques investigated here.

\section{Discussion}

The performance of the different spectral estimation methods as well as the definition of an optimal solution have to be considered in the frame of this study, i.e., for 3-D reconstruction of complex urban environments using a minimal number of images. To this end, a case study of a dense urban area imaged by the DLR's E-SAR sensor in a fully polarimetric mode, i.e., $N_{p}=3$ at L-band with $M=3$ images is investigated. This application data set was acquired on August 1, 2000, with a small baseline of $10 \mathrm{~m}$ and a large one of $40 \mathrm{~m}$. The SAR images have a resolution of $2 \mathrm{~m}$ in range, and $3 \mathrm{~m}$ in azimuth.

1) Coherent Scattering Behavior: The diversity of statistical scattering behavior over the observed scene may be appreciated in the light of the results of the coherent Time-frequency (TF) analysis led in [20] using a single PolSAR image. The correlation indicator $\rho_{\mathrm{TF}}$ shown in Fig. 5 and derived in [20] and [46] shows that coherent scatterers, with $\rho_{\mathrm{TF}}$ close to 1 , mostly correspond with built-up structures, whereas distributed scatterers, whose $\rho_{\mathrm{TF}}$ tends to equal 0 , indicate vegetated areas, bare surface and shadow areas. However, over many regions of the scene, $\rho_{\mathrm{TF}}$ takes intermediate values, characteristic of hybrid scattering environments, as they were defined in [20]. This observation reveals the important need for adaptive tomographic techniques, able to deal with different types of statistical scattering behaviors, encountered over such complex environments as urban areas. Adaptivity being an intrinsic feature of WSF techniques, their use over dense builtup areas presents great potential.

2) Comparison of Single Polarization Tomographic Techniques: A comparison between WSF techniques and other commonly used single polarization tomographic estimators is given using the Dresden test data set. Tomograms are computed at VV polarization, along the path aligned with the range direction, passing by two buildings, and indicated in yellow in Fig. 6. Here, the model order is uniformly fixed at $\hat{n}_{s}=2$ and tomograms are computed in slant range, in order to demonstrate the performance of tomographic estimators for sidelobe suppression and robustness against model order mismatch. As shown in Fig. 7(a) and (b), the nonparametric approaches BF and Capon permit to localize some dominant scattering sources, but are severely limited by their coarse vertical resolution, and they cannot precisely estimate the different features of buildings. The HR capability for scatterers' separation of the evaluated parametric techniques is demonstrated in Fig. 7(c)-(f). Nevertheless, the different methods investigated in this assessment do not show similar levels of discrimination and robustness. As indicated in Fig. 7 using magenta ellipses, sidelobes over the ground may be observed in the tomograms computed using MUSIC, DML, and NSF techniques. These artifacts are due to model order mismatch, whereas those observed over buildings are either due to irregularly sampled baselines, or to the limitations of these approaches with respect to source correlation. One may 


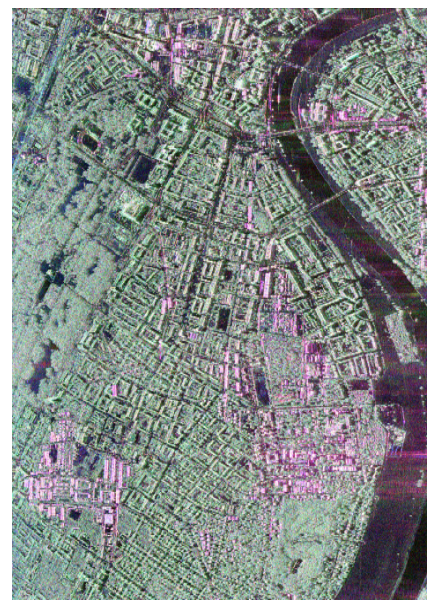

(a)

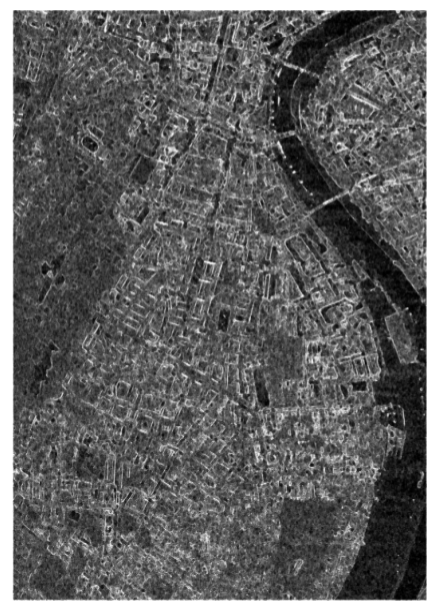

(b)
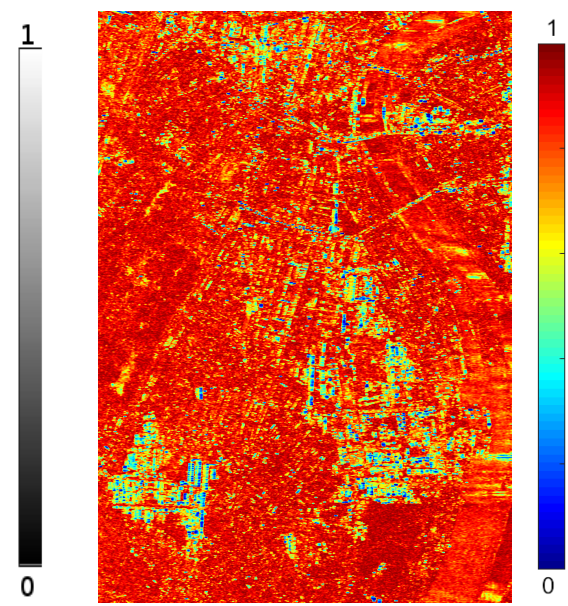

(c)

Fig. 5. Polarimetric features of the intermediate resolution L-band SAR data acquired over the city of Dresden [20]. (a) Pauli-coded image. (b) TF correlation $\rho_{\mathrm{TF}}$. (c) PolSAR Entropy $H$.

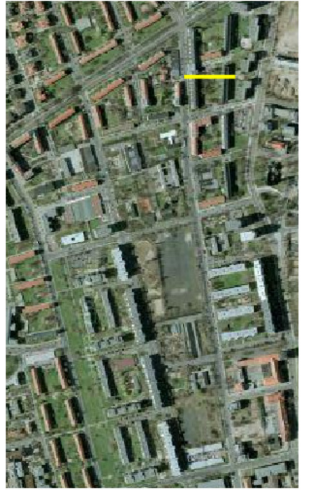

(a)

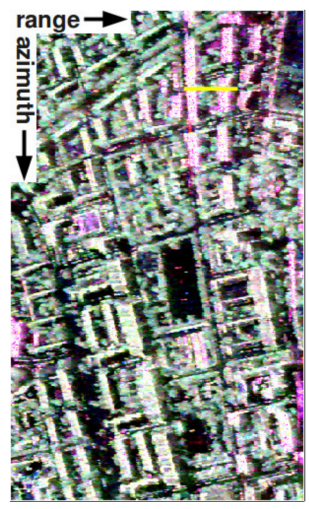

(b)
Fig. 6. Selected test area and tomographic profile location. (a) Google image(c). (b) Pauli-coded image.

observe that results obtained with the SSF technique and given in Fig. 7(f) show great robustness for sidelobe suppression even in this extreme case. The superiority of SSF and DML approaches with respect to MUSIC and NSF in the presence of strong source correlation is due to the rank deficiency of the source covariance matrix [38], [47]. In such a case, the true steering vectors are not all orthogonal to the noise subspace [47] and some sources simply cannot be discriminated. This limitation may be overcome by working on the signal subspace that is complementary to the noise subspace. Theoretical demonstrations and numerical illustrations may be found in [38], indicating that the NSF estimator is asymptotically equivalent to the SSF one when dealing with uncorrelated signals, but is less efficient for the estimation of coherent or highly correlated signals. A correct model order estimate is required to obtain the optimal weighting matrix of NSF, so that this estimator can achieve its optimal performance. In the case of completely correlated sources, the first eigenvector of the data covariance matrix is composed of linear combinations of the steering vectors of all sources, and the SSF and DML approaches have to face a very similar problem, that consists of estimating structured steering vectors from their weighted sum. One may note that in the case of uncorrelated source signals, MUSIC has a noticeably good performance, as it is asymptotically equivalent to the DML. As stated in [41], one may theoretically show that the optimally weighted SSF never performs worse than DML and, in general, outperforms DML. The difference between performance can be large in the case of highly correlated source signals [41]. Finally, according to the theoretical study proposed in [29], it turns out that the DML estimator is not statistically efficient if the number of images, $M$, is small, even if the number of looks used to perform the estimation, $L$, is large. DML estimates can achieve the CRLB only if $M$ is takes sufficiently large values. In the context of tomographic reconstruction, one may understand this limitation as the difficulty to estimate linear combinations of structured vectors using a small set of $M$ observations. For larger $M$ values, the improved level of sparsity, with $M \gg \hat{n}_{s}$, permits the DML approach to reach high performance. This is basically what is done in the so-called compressive-sensing approaches presented in [2] and [3], where a $L_{1}$ norm constraint is added to the DML criterion in order to select the model order.

3) Polarimetric Information Handling: In the extreme configuration studied in this work, it is obvious that polarization diversity may play an important role in the discrimination of closely spaced sources, as previously reported in [18], [4], [15], and [48]. Nevertheless, as shown in Fig. 4, the performance of these techniques may be modulated by the randomness of the observed polarimetric information. Moreover, the 2-D entropy, $H$, computed over the test area and displayed in Fig. 5(c), reaches high values over the studied areas, indicating the limited possibilities of 2-D SAR polarimetry for characterizing this region. PolTomoSAR techniques are expected to overcome these limitations by discriminating contributions merged in layover areas and effectively estimate the dominant scattering patterns. Nevertheless, over complex media or 3-D structures containing numerous different scatterers into vertical sections smaller than the tomographic vertical 


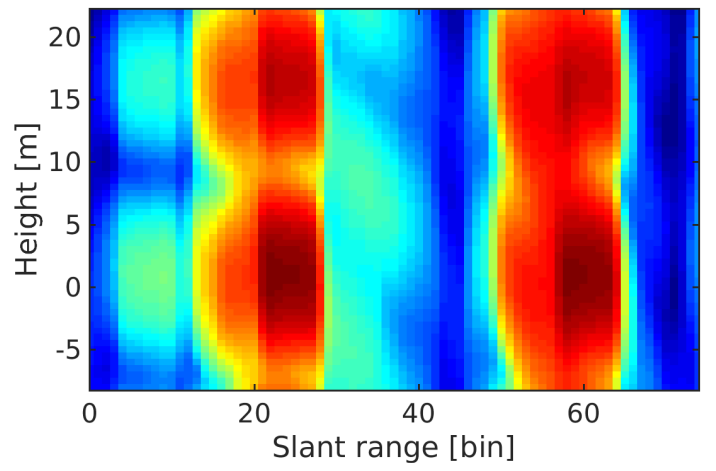

(a)

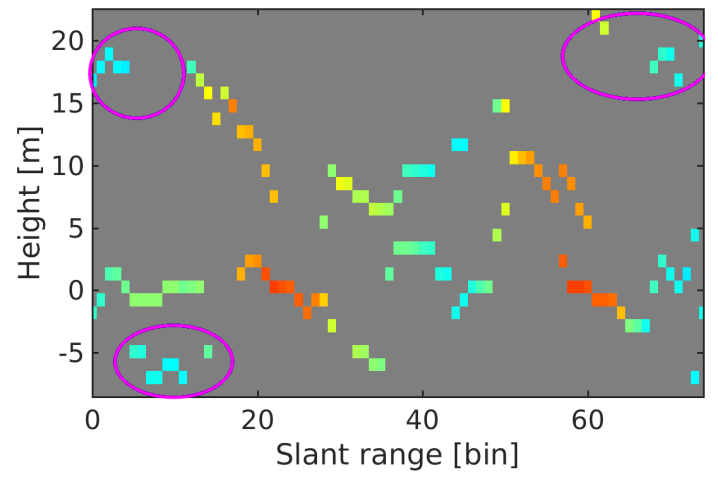

(c)

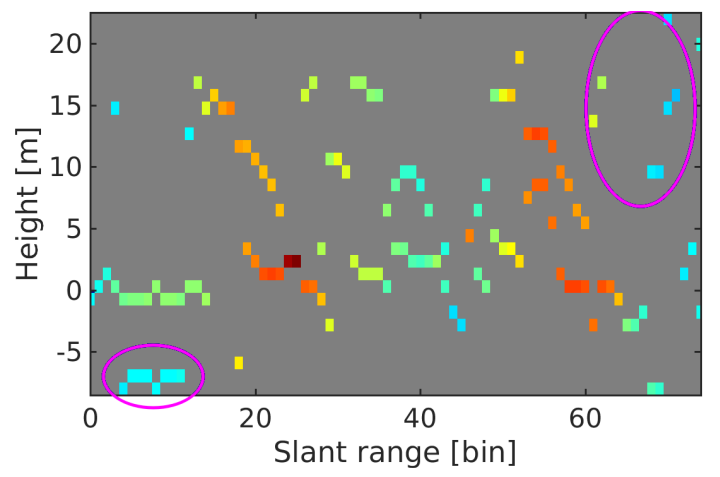

(e)

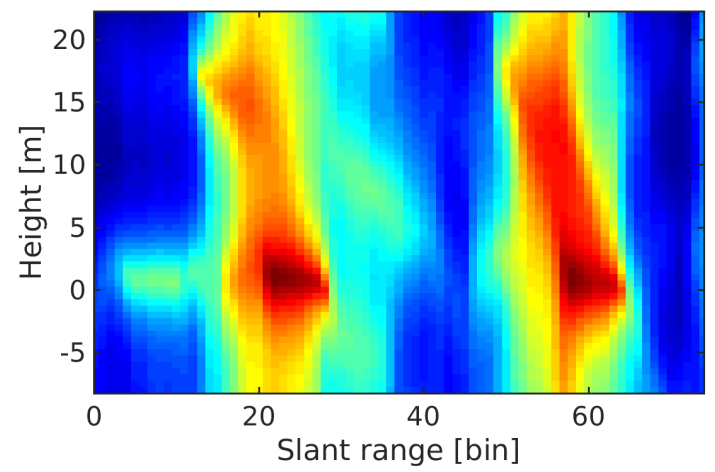

(b)

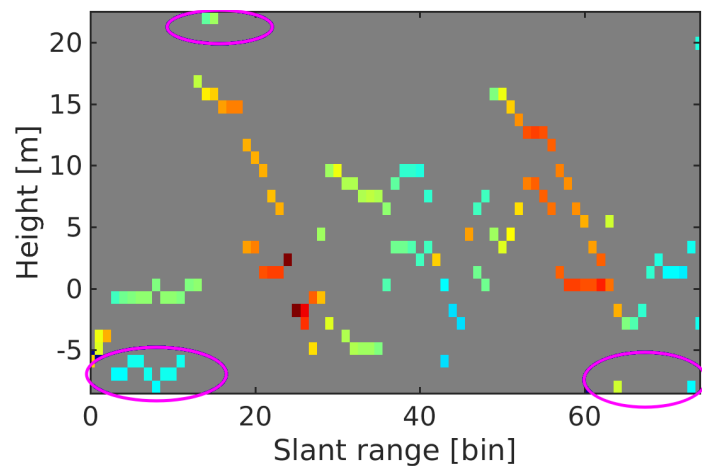

(d)

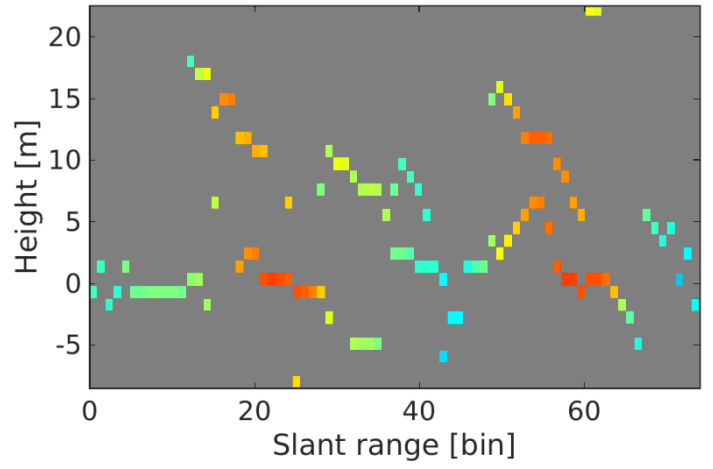

(f)

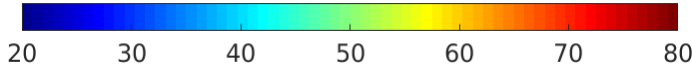

Fig. 7. VV reflectivity tomograms (in $\mathrm{dB}$ ) estimated in slant range along the profile shown in Fig. 6 . The area without scattering response is indicated in gray color. (a) Beamforming. (b) Capon. (c) MUSIC $\left(\hat{n}_{s}=2\right)$. (d) DML $\left(\hat{n}_{s}=2\right)$. (e) $\operatorname{NSF}\left(\hat{n}_{s}=2\right)$. (f) $\operatorname{SSF}\left(\hat{n}_{s}=2\right)$.

resolution, one may still observe high entropy values. In such cases, classical unitary-rank tomographic techniques are not adapted and may lead to useless indicators. We then propose to resort to FR polarimetric techniques, such as the ones proposed in [21], which can provide second-order polarimetric features, i.e., polarimetric covariance matrices, in 3-D. The outputs of this technique may then be processed using existing techniques, formerly developed for 2-D applications, and extended to 3-D analysis.

4) Required Features for Tomographic Focusing in a Minimal Configuration: From the elements of the discussion presented above, it is possible to define some key properties an estimator should possess in order to perform tomographic focusing in a minimal configuration with a satisfying level of quality. HR capabilities and coherent source, i.e., statistical diversity, handling are among the most required features, as shown by SP experiments over real data. Polarimetric information shall be used to improve the estimation results. From the experimental and theoretical properties mentioned previously, two methods, the SSF and DML, seem to satisfy all these requirements, and their extension to the polarimetric case is the subject of Section III.

\section{PROPOSED POLARIMETRIC SSF AND DML ESTIMATORS}

This section presents a solution to HR polarimetric tomographic focusing on the P-SSF and P-DML approaches. A generic unitary-rank polarimetric optimization technique,

\section{ACCEPTED MANUSCRIPT}


based on the extension of the alternating projection approach [49] to the polarimetric case is first proposed. This method is then adapted to the P-SSF and P-DML criteria.

\section{A. Polarimetric Optimization Using Alternating Projections}

The optimization of both the P-SSF and P-DML criteria may be cast as

$$
\widehat{\mathbf{z}}, \widehat{\mathbf{K}}=\arg \max _{\mathbf{z}, \mathbf{K}} Q(\mathbf{z}, \mathbf{K})
$$

with

$$
Q(\mathbf{z}, \mathbf{K})=\operatorname{tr}\left(\mathbf{P}_{\left.\mathbf{A}_{(\mathbf{z}, \mathbf{K})} \mathbf{M}\right)}\right.
$$

where $\mathbf{M}$ is a $\left(N_{P} M \times N_{P} M\right)$ positive semidefinite hermitian matrix that will be, respectively, defined by the P" SSF and P" DML estimator, and $\mathbf{P}_{\mathbf{A}_{(z, K)}}$ represents the projector onto the space spanned by the columns of $\mathbf{A}_{(\mathbf{z}, \mathbf{K})}$, given by

$$
\mathbf{P}_{\mathbf{A}}=\mathbf{A}\left(\mathbf{A}^{H} \mathbf{A}\right)^{-1} \mathbf{A}^{H}
$$

where the arguments $(\mathbf{z}, \mathbf{K})$ have been omitted, and with $\mathbf{A}=\left[\mathbf{a}\left(z_{1}, \mathbf{k}_{1}\right), \ldots, \mathbf{a}\left(z_{n_{s}}, \mathbf{k}_{n_{s}}\right)\right]$ the $N_{P} M \times n_{s}$ PolTomoSAR steering matrix. Using the fact that $\mathbf{P}_{\mathbf{A}}$ remains invariant by any permutation of the columns of $\mathbf{A}$, one may rewrite the projector as $\mathbf{P}_{\mathbf{A}}=\mathbf{P}_{\left[\tilde{\mathbf{A}}_{i} \mathbf{a}\left(z_{i}, \mathbf{k}_{i}\right)\right]}$, with

$$
\widetilde{\mathbf{A}}_{i}=\left[\mathbf{a}_{1}, \ldots, \mathbf{a}_{i-1}, \mathbf{a}_{i+1}, \ldots, \mathbf{a}_{n_{s}}\right.
$$

where the notation $\mathbf{a}_{i}=\mathbf{a}\left(z_{i}, \mathbf{k}_{i}\right)$ is adopted. This expression may then be used to optimize the criterion in (7) with respect to a single source at a time. This mono-dimensional approach writes

$$
\hat{z}_{i}, \widehat{\mathbf{k}}_{i}=\arg \max _{z, \mathbf{k}} Q_{i}(z, \mathbf{k})
$$

with

$$
Q_{i}(z, \mathbf{k})=\operatorname{tr}\left(\mathbf{P}_{\left[\tilde{\mathbf{A}}_{i} \mathbf{a}(z, \mathbf{k})\right]} \mathbf{M}\right) .
$$

The maximization of (7) may then be achieved by alternatively optimizing the 1-D criterion (10) for different values of $i \in\left[1, \ldots, n_{s}\right]$, until convergence. As reported in [49] in the SP case, despite its suboptimal character, this method generally shows a satisfying convergence behavior, provided that it is initialized in an adequate way. Its main interest resides in the way the optimization in (10) can be conducted. The projector of a compound matrix verifies the following expression:

$$
\mathbf{P}_{\left[\widetilde{\mathbf{A}}_{i} \mathbf{a}(z, \mathbf{k})\right]}=\mathbf{P}_{\widetilde{\mathbf{A}}_{i}}+\mathbf{P}_{\mathbf{a}(z, \mathbf{k}) \tilde{\mathbf{A}}_{i}}
$$

with

$$
\mathbf{a}(z, \mathbf{k})_{\widetilde{\mathbf{A}}_{i}}=\mathbf{P}_{\widetilde{\mathbf{A}}_{i}}^{\perp} \mathbf{a}(z, \mathbf{k})=\mathbf{b}(z, \mathbf{k})
$$

where $\mathbf{P}_{\widetilde{\mathbf{A}}_{i}}^{\perp}=\left(\mathbf{I}-\mathbf{P}_{\widetilde{\mathbf{A}}_{i}}\right)$, and is the result of the projection of the considered steering vector onto the space orthogonal to the one spanned by the reduced matrix $\widetilde{\mathbf{A}}_{i}$. Taking into account that $\mathbf{P}_{\mathbf{b}}=\mathbf{b b}^{H} /\left(\mathbf{b}^{H} \mathbf{b}\right)$, the criterion in (10) may be rewritten as

$$
Q_{i}(z, \mathbf{k})=\operatorname{tr}\left(\mathbf{P}_{\widetilde{\mathbf{A}}_{i}} \mathbf{M}\right)+\frac{\mathbf{b}^{H}(z, \mathbf{k}) \mathbf{M b}(z, \mathbf{k})}{\|\mathbf{b}(z, \mathbf{k})\|^{2}}
$$

with $\mathbf{b}(z, \mathbf{k})$ defined in (11). One may note that the first term of the right-hand side of (12) does not depend on the parameters used to optimize the criterion, $z$ and $\mathbf{k}$ and can then be dropped. The single source optimization of (10) can then be simplified as

$$
\begin{aligned}
\hat{z}_{i}, \widehat{\mathbf{k}}_{i} & =\arg \max _{z, \mathbf{k}} \frac{\mathbf{b}^{H}(z, \mathbf{k}) \mathbf{M b}(z, \mathbf{k})}{\|\mathbf{b}(z, \mathbf{k})\|^{2}} \\
& =\arg \max _{z, \mathbf{k}} \widetilde{Q}_{i}(z, \mathbf{k}) .
\end{aligned}
$$

By replacing $\mathbf{b}(z, \mathbf{k})$ with $\mathbf{b}(z)$ in (13), one gets the SP expression derived in [49], which may be assimilated to a $\mathrm{BF}$ result, normalized by $\|\mathbf{b}(z)\|^{2}$, and evaluated at very low computational cost. In the polarimetric case, one cannot, as in the classical polarimetric mono-dimensional case, i.e., using P-CAPON and P-MUSIC, directly optimize (13) using an eigendecomposition, since $\|\mathbf{b}(z, \mathbf{k})\|^{2}$ cannot be determined a priori, $\mathbf{k}$ being unknown. By inserting the alternative expression of a PolTomoSAR steering vector of (4) into (11), one gets

$$
\mathbf{b}(z, \mathbf{k})=\mathbf{P}_{\widetilde{\mathbf{A}}_{i}}^{\perp} \mathbf{B}_{\mathbf{a}}(z) \mathbf{k}=\mathbf{B}_{\mathbf{a}}(z)_{\widehat{\mathbf{A}}_{i}} \mathbf{k}
$$

the criterion in (13) can be rewritten as

$$
\widetilde{Q}_{i}(z, \mathbf{k})=\frac{\mathbf{k}^{H} \mathbf{M}^{\prime}(z) \mathbf{k}}{\mathbf{k}^{H} \mathbf{B}^{\prime}(z) \mathbf{k}}
$$

with

$$
\begin{aligned}
\mathbf{M}^{\prime}(z) & =\mathbf{B}_{\mathbf{a}}{ }^{H}(z)_{\widehat{\mathbf{A}}_{i}} \mathbf{M B}_{\mathbf{a}}(z)_{\widehat{\mathbf{A}}_{i}} \\
\mathbf{B}^{\prime}(z) & =\mathbf{B}_{\mathbf{a}}{ }^{H}(z)_{\widehat{\mathbf{A}}_{i}} \mathbf{B}_{\mathbf{a}}(z)_{\widehat{\mathbf{A}}_{i}}
\end{aligned}
$$

where the $\left(N_{P} \times N_{P}\right)$ matrix $\mathbf{M}^{\prime}(z)$ may be associated with a version of $\mathbf{M}$ focused at the elevation position $z$. Given that both $\mathbf{M}^{\prime}(z)$ and $\mathbf{B}^{\prime}(z)$ are positive semidefinite hermitian matrices, the maximization of (15) may be achieved through the following generalized eigenvalue decomposition:

$$
\mathbf{M}^{\prime}(z) \mathbf{k}=\lambda \mathbf{B}^{\prime}(z) \mathbf{k} \quad \text { s.t. } \quad \mathbf{k}^{H} \mathbf{k}=1
$$

where the generalized eigenvalues represent optimal solutions for $\widetilde{Q}_{i}(z, \mathbf{k})$, reached for scattering vectors given by the corresponding eigenvectors. Therefore, at a given iteration of the P-AP algorithm, the parameters of the $i^{t h}$ scatterer are estimated as

$$
\begin{aligned}
\hat{z}_{i} & =\arg \max _{z} \lambda_{\max }\left(\mathbf{M}^{\prime}(z), \mathbf{B}^{\prime}(z)\right) \\
\widehat{\mathbf{k}}_{i} & =\mathbf{e}_{\max }\left(\mathbf{M}^{\prime}\left(\hat{z}_{i}\right), \mathbf{B}^{\prime}\left(\hat{z}_{i}\right)\right)
\end{aligned}
$$

where $\lambda_{\max }(\mathbf{A}, \mathbf{B}) \geq 0$, represents the largest generalized eigenvalue of $\mathbf{A}, \mathbf{B} \geq \mathbf{0}$, and $\mathbf{e}_{\max }(\mathbf{A}, \mathbf{B})$ is the corresponding eigenvector. One may note here that the evaluation of the orthogonal projector and the generalized eigenvalue problem required in (17) shall be conducted with care in order to ensure that the whole approach provides meaningful results. Indeed, orthogonal projectors are generally not evaluated directly through (8), but rather using more stable procedures, based on matrix decompositions, such as the QR factorization [38]. Reducing the generalized eigenvalue decomposition in (16) to a classical eigenvalue problem through matrix inversion, or pseudo inversion, should, in a general way, be avoided, since such an alternative may break conditions for hermitian symmetry, and hence lead to meaningless results. The synopsis 


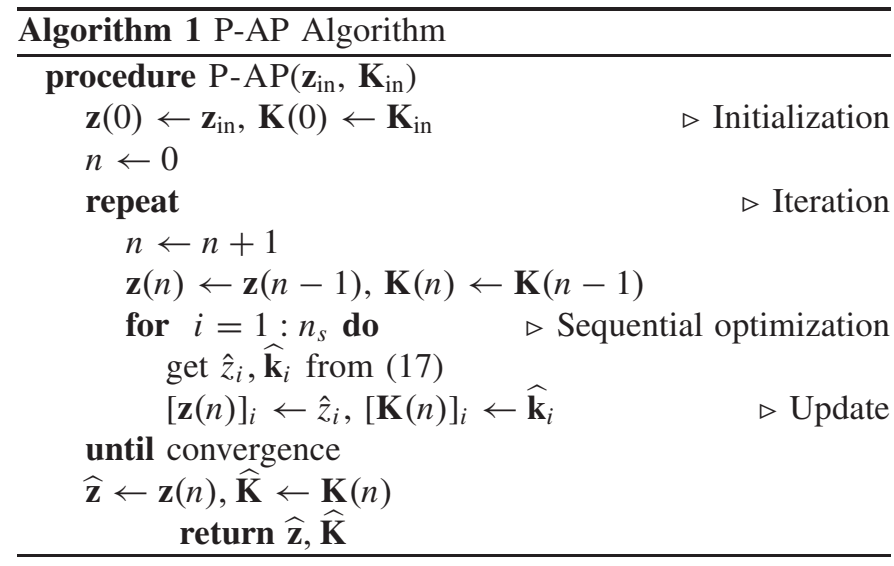

of the proposed P-AP algorithm is given in Algorithm 1. As mentioned earlier, the algorithm shall be initialized with good enough parameter estimates, such as those provided by the P-MUSIC 1-D algorithm, for instance. The convergence of this technique may be decided by evaluating the amount of change from one iteration to the other, of one or several indicators, such as the criterion to be optimized $Q(\mathbf{z}, \mathbf{K})$, or its parameters $\mathbf{z}, \mathbf{K}$. Dealing with these parameters might help to set a reasonable practical threshold of convergence.

\section{B. Polarimetric SSF Estimator}

Weighted subspace-based spectral estimation techniques were proposed in [41] and [38], and rely on the eigendecomposition of the covariance matrix of the measured signal. In the PolTomoSAR case, this covariance may be written as

$$
\begin{aligned}
\mathbf{R} & =\mathbf{A}(\mathbf{z}, \mathbf{K}) \mathbf{R}_{\mathbf{s s}} \mathbf{A}^{H}(\mathbf{z}, \mathbf{K})+\sigma_{n}^{2} \mathbf{I} \\
& =\mathbf{E} \boldsymbol{\Lambda} \mathbf{E}^{H}=\mathbf{E}_{s} \boldsymbol{\Lambda}_{s} \mathbf{E}_{s}^{H}+\sigma_{n}^{2} \mathbf{E}_{n} \mathbf{E}_{n}^{H}
\end{aligned}
$$

where $\boldsymbol{\Lambda}=\operatorname{diag}\left(\left\{\lambda_{i}\right\}_{i=1}^{N_{P} M}\right)$, with $\lambda_{i} \geq \lambda_{i+1} \geq 0$, denotes the eigenvalue matrix of $\mathbf{R}$, and $\mathbf{E}$ is its corresponding orthonormal eigenvector matrix. The PolTomoSAR responses of $n_{s}$ scatterers occupy a subspace whose dimension is at most $n_{s}$, the remaining eigenvalues of $\mathbf{R}$ being equal to the noise variance, i.e., $\left\{\lambda_{i}\right\}_{i=n_{s}+1}^{N_{P} M}=\sigma^{2}$. One may then define the so-called signal subspace by $\mathbf{E}_{s}=\left[\mathbf{e}_{1}, \ldots, \mathbf{e}_{n_{s}}\right]$ with eigenvalues $\boldsymbol{\Lambda}_{s}=\operatorname{diag}\left(\left[\lambda_{1}, \ldots, \lambda_{n_{s}}\right]\right)$, whereas the noise subspace is given by $\mathbf{E}_{n}=\left[\mathbf{e}_{n_{s}+1}, \ldots, \mathbf{e}_{N_{P} M}\right]$. It is clear from (18) that if the source covariance matrix, $\mathbf{R}_{\mathbf{s s}}$, has rank $n_{s}$, then $\mathbf{E}_{n}$ is orthogonal to $\mathbf{A} \mathbf{R}_{\text {ss }} \mathbf{A}^{H}$, implying that the space range of $\mathbf{E}_{s}$ coincides with the one of $\mathbf{A}(\mathbf{z}, \mathbf{K})$. These properties may be exploited to estimate signal parameters using weighted LS fitting criteria, defined as

$$
\begin{aligned}
& Q_{\text {P-NSF }}(\mathbf{z}, \mathbf{K})=\left\|\widehat{\mathbf{E}}_{n}^{H} \mathbf{A}(\mathbf{z}, \mathbf{K})\right\|_{\mathbf{W}}^{2} \\
& Q_{\mathrm{P}-\mathrm{SSF}}(\mathbf{z}, \mathbf{K})=\left\|\widehat{\mathbf{E}}_{s}-\mathbf{A}(\mathbf{z}, \mathbf{K}) \mathbf{T}\right\|_{\mathbf{W}}^{2}
\end{aligned}
$$

where $\|\mathbf{X}\|_{\mathbf{W}}^{2}=\operatorname{tr}\left(\mathbf{X W} \mathbf{X}^{H}\right)$. The use of a weighting matrix, $\mathbf{W}$, aims to compensate or attenuate the effects of discrepancies in the relationship between subspaces and measured signals, when computations are led from a covariance matrix estimated with a finite number of looks, i.e., when using $\widehat{\mathbf{R}}, \widehat{\mathbf{E}}, \widehat{\mathbf{\Lambda}}$, in place of $\mathbf{R}, \mathbf{E}, \boldsymbol{\Lambda}$. The different solutions obtained for
WSF estimators are presented in details in [41]. After replacing in (19) $\mathbf{T}$ by it LS estimate $\widehat{\mathbf{T}}=\mathbf{A}^{\dagger}(\mathbf{z}) \widehat{\mathbf{E}}_{s}, \mathbf{X}^{\dagger}$ being the pseudo-inverse of $\mathbf{X}$, the P-SSF optimization may be written as

$$
\widehat{\mathbf{z}}, \widehat{\mathbf{K}}=\arg \max _{\mathbf{z}, \mathbf{K}} \widetilde{Q}_{\mathrm{P}-\mathrm{SSF}}(\mathbf{z}, \mathbf{K})
$$

with

$$
\begin{aligned}
\widetilde{Q}_{\mathrm{P}-\mathrm{SSF}}(\mathbf{z}, \mathbf{K}) & =\operatorname{tr}\left(\mathbf{P}_{\mathbf{A}(\mathbf{z}, \mathbf{K})} \mathbf{M}\right) \\
\mathbf{M} & =\widehat{\mathbf{E}}_{s} \mathbf{W} \widehat{\mathbf{E}}_{s}^{H} .
\end{aligned}
$$

This optimization is similar to the generic one introduced in (7), and hence, it may be conducted efficiently using the P-AP algorithm depicted in Algorithm 1. Depending on the weighting matrix selected in (19), one may obtain tomographic estimators with different properties. Inserting $\mathbf{W}=\left(\mathbf{A}^{H} \hat{\mathbf{E}}_{s}\left(\widehat{\boldsymbol{\Lambda}}_{s}-\hat{\sigma}^{2} \mathbf{I}\right)^{-2} \widehat{\boldsymbol{\Lambda}}_{s} \hat{\mathbf{E}}_{s}^{H} \mathbf{A}\right)^{-1}$ into the cost function $Q_{\mathrm{P}-\mathrm{NSF}}(\mathbf{z}, \mathbf{K})$ yields the optimal P-NSF estimator, whose estimation error covariance attains the CRLB [19], [41]. The equivalent optimal P-SSF estimator is obtained by incorporating $\mathbf{W}=\left(\widehat{\boldsymbol{\Lambda}}_{s}-\hat{\sigma}^{2} \mathbf{I}\right)^{2} \widehat{\boldsymbol{\Lambda}}_{s}^{-1}$ in $Q_{\mathrm{P}-\mathrm{SSF}}(\mathbf{z}, \mathbf{K})$ [38]. These optimal WSF estimators are used in this article. Once the elevation and polarimetric features of different scatterers are determined, one may estimate their reflectivity using a classical approach such as the minimization of an LS criterion

$$
\widehat{\sigma}_{i}=\frac{1}{L} \sum_{l=1}^{L}\left|\widehat{s}_{i}(l)\right|^{2} \text {, with } \widehat{\mathbf{s}}(l)=\mathbf{A}^{\dagger}(\widehat{\mathbf{z}}, \widehat{\mathbf{K}}) \mathbf{y}(l) .
$$

\section{Polarimetric DML Estimator}

From the Gaussian statistical behavior given under the CM model assumption, one may derive the likelihood of the $L$ acquired independent signals, $\{\mathbf{y}(l)\}_{l=1}^{L}$. As shown in [38], reflectivities can be estimated using the LS approach described in (21), and the DML optimization is written as

$$
\widehat{\mathbf{z}}, \widehat{\mathbf{K}}=\arg \max _{\mathbf{z}, \mathbf{K}} Q_{\text {P-DML }}(\mathbf{z}, \mathbf{K})
$$

with

$$
\widetilde{Q}_{\text {P-DML }}(\mathbf{z}, \mathbf{K})=\operatorname{tr}\left(\mathbf{P}_{\mathbf{A}(\mathbf{z}, \mathbf{K})} \widehat{\mathbf{R}}\right) .
$$

Similar to the P-SSF case, the optimization described in (22) may be achieved efficiently using the P-AP algorithm given in Algorithm 1, and replacing $\mathbf{M}$ with $\widehat{\mathbf{R}}$ in (7).

\section{Illustration of the Performance of the P-SSF and P-DML Estimators}

Two examples are simulated in order to illustrate the performance of the proposed P-SSF and P-DML estimators, in the presence of coherent scatterers, and for low SNR values. The received MB-PolInSAR signals from two coherent scatterers with $\Delta z \in[0,4] \mathrm{m}$ or SNR $\in[0,30] \mathrm{dB}$, are simulated in two polarimetric cases, $\mathbf{k}_{1} \| \mathbf{k}_{2}$, and $\mathbf{k}_{1} \perp \mathbf{k}_{2}, \mathbf{k}_{i}(i=1,2)$ being chosen arbitrarily. 


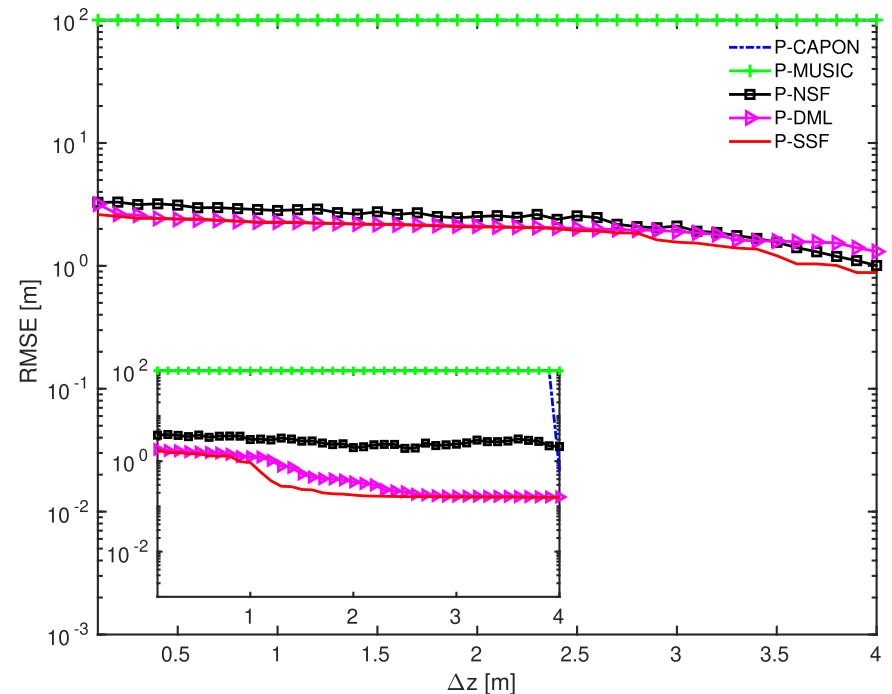

(a)

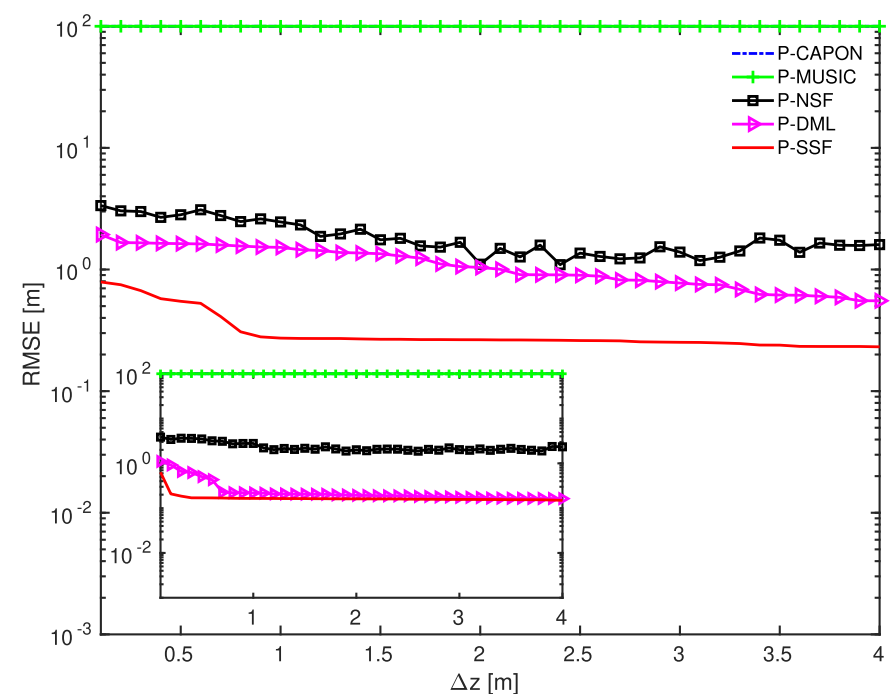

(b)

Fig. 8. Height RMSE of estimation performance of unitary-rank polarimetric estimators for a varying height difference $\Delta z . \rho=0.995, \mathrm{SNR}=0 \mathrm{~dB}$. (Outer Plot) $M=3$. (Inner Plot) $M=6$ (a) $\mathbf{k}_{1} \| \mathbf{k}_{2}$. (b) $\mathbf{k}_{1} \perp \mathbf{k}_{2}$.

1) Estimation Accuracy at Low SNR and High Correlation: This example aims to evaluate the estimation accuracy for coherent scatterers $(\rho=0.995)$ using unitary-rank polarimetric tomographic methods in the low-SNR case, i.e., SNR $=0 \mathrm{~dB}$. In Fig. 8, P-MUSIC and P-Capon techniques show a low performance, whatever the separation in elevation $\Delta z$, due to the rank deficiency of $\mathbf{R}_{\mathrm{ss}}$. When $\mathbf{k}_{1} \| \mathbf{k}_{2}$ [Fig. 8(a)], in the case of $M=6$, the performance of P-DML technique degrades for $\Delta z \leq 2.5 \mathrm{~m}$ but P-SSF maintains a low RMSE for extremely small $\Delta z$ values. In the very low resolution case, $M=3$, all the methods show a similar behavior, with an RMSE of $1-2 \mathrm{~m}$ over the whole studied range of $\Delta z$. In the case of a favorable polarimetric diversity, $\mathbf{k}_{1} \perp \mathbf{k}_{2}$, the P-SSF polarimetric adaptivity leads to a significant improvement for the separation of closely spaced scatterers, as shown in Fig. 8(b). In summary, the PSSF technique shows a performance equal to, or much higher than, one of the P-DML approach.

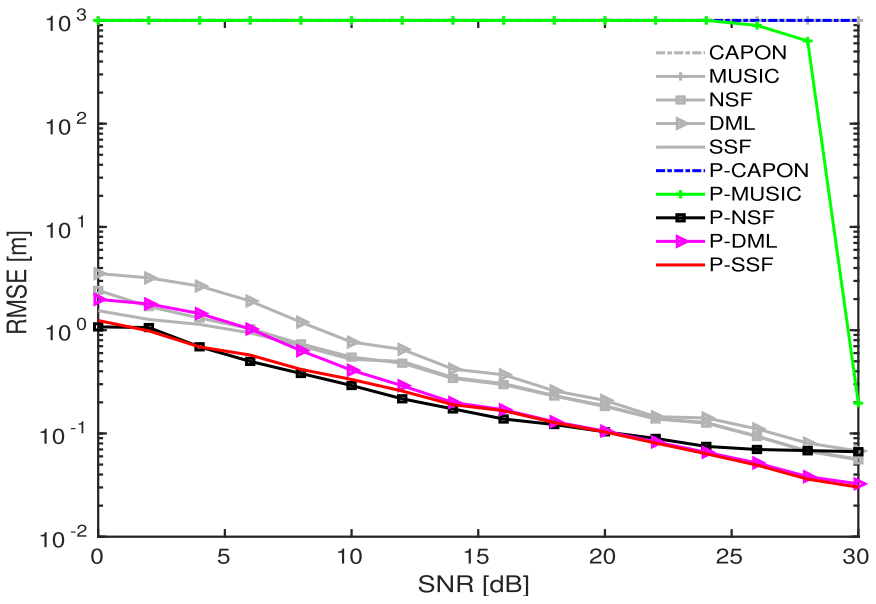

(a)

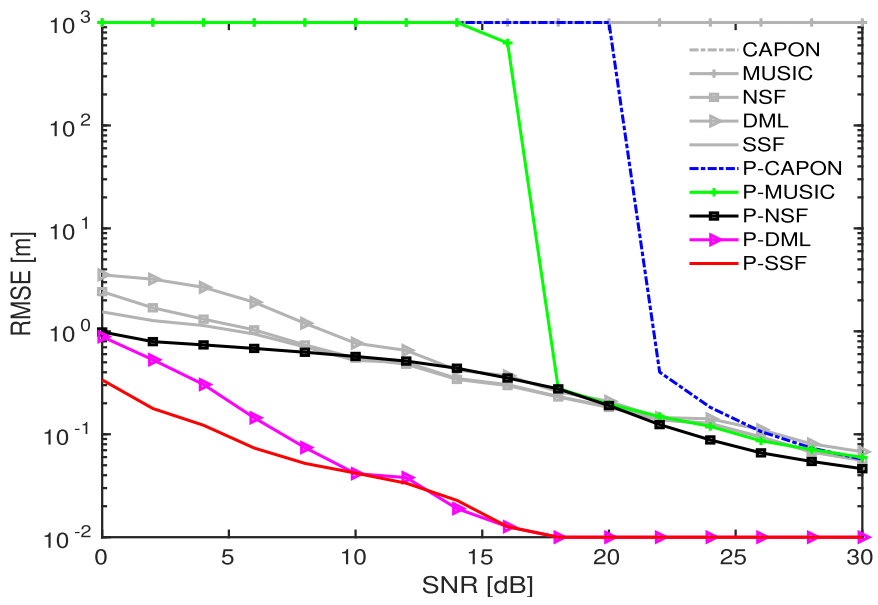

(b)

Fig. 9. Height RMSE of unitary-rank polarimetric estimators with varying SNR values, $\rho=0.995$. Results of SP estimators are shown in gray. (a) $M=3, \mathbf{k}_{1} \| \mathbf{k}_{2}$. (b) $M=3, \mathbf{k}_{1} \perp \mathbf{k}_{2}$.

2) SNR Requirement for Accurate Estimation: The different polarimetric tomographic estimators are applied to signals simulated for two CM scatterers, with $\rho=0.995$ and $\Delta z=4 \mathrm{~m}$ observed at varying SNR values. As one can observe in Fig. 9, multidimensional polarimetric estimators perform much better than the mono-dimensional P-MUSIC and P-Capon techniques. However, the results of the P-NSF technique are often worse than those of the P-DML and P-SSF techniques, due to an inaccurate estimation of the noise subspace in the presence of coherent scatterers. Compared with the P-DML technique, the gain in performance of the P-SSF technique is more prominent in the low-SNR case $(\mathrm{SNR}<10 \mathrm{~dB})$ than in the high-SNR case. Among all the methods investigated in this study, the P-SSF technique maintains the lowest RMSE and demonstrates overall robust performance. The use of parallel scattering vectors leads to results similar to those obtained in the single polarization case, as shown using gray curves in Fig. 9(a).

\section{E. Application}

In the extreme case of $M=3$ acquisitions, several techniques are applied to estimate Digital Surface Models (DSMs)

\section{ACCEPTED MANUSCRIPT}




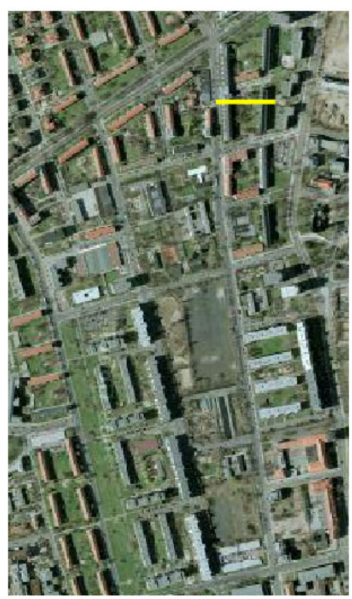

(a)

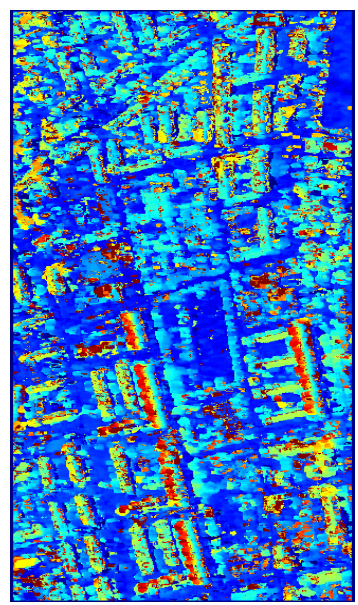

(d)

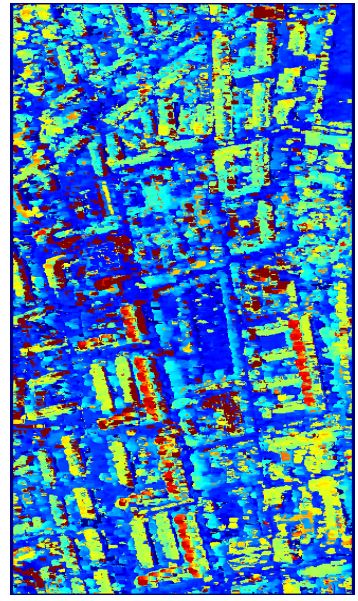

(g)

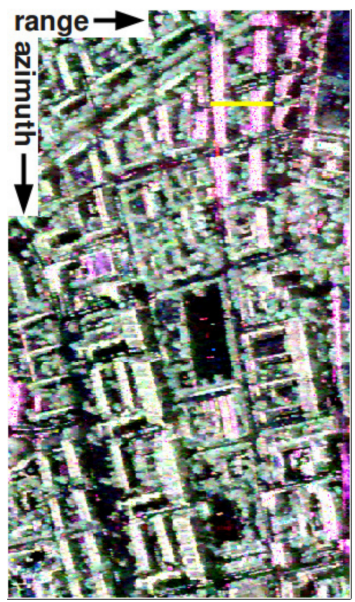

(b)

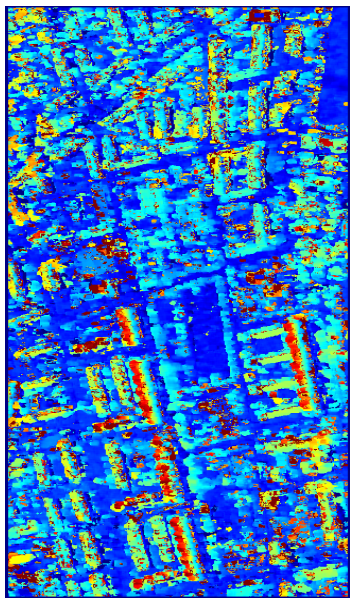

(e)

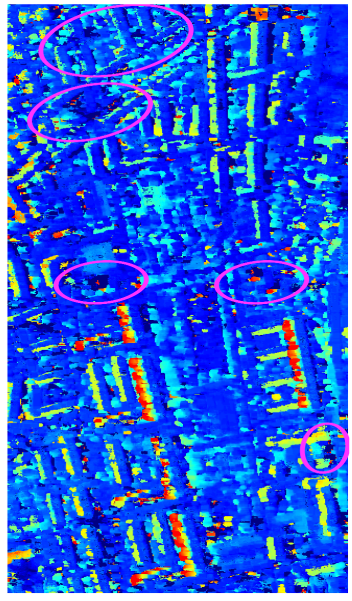

(h)

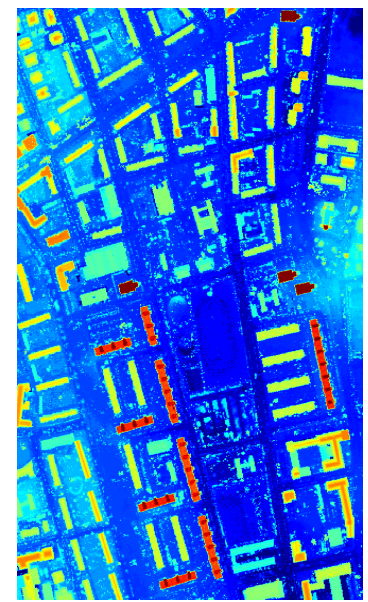

(c)

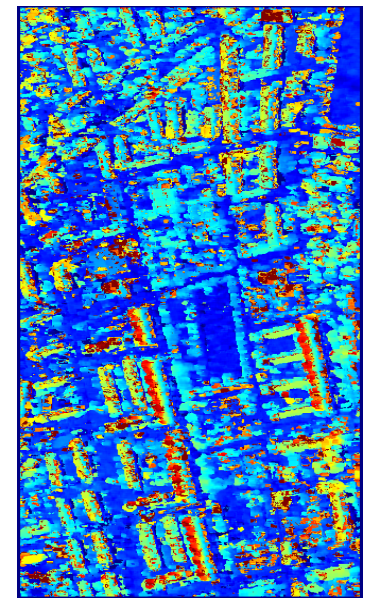

(f)

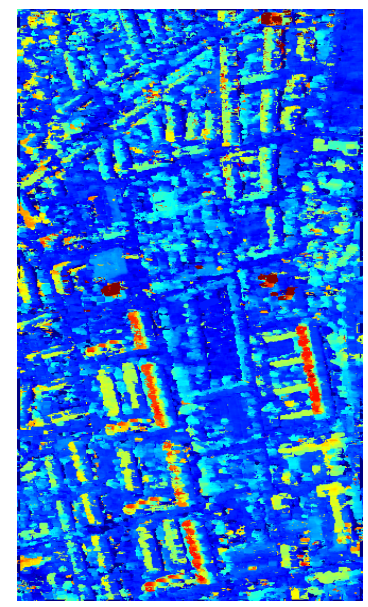

(i)

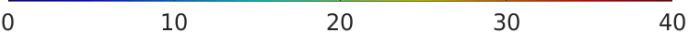

Fig. 10. DSMs (in meter) derived from polarimetric SAR data stack using single-polarization and polarimetric MUSIC and WSF estimators, $\hat{n}_{s}=2$, compared to the LiDAR-derived DSM. (a) Google image. (b) Pauli-coded image. (c) LiDAR. (d) VV MUSIC. (e) VV NSF. (f) VV SSF. (g) P-MUSIC. (h) P-NSF. (i) P-SSF.

over the whole test area, which are compared with a LiDARderived 3-D map, as shown in Fig. 10. The LiDAR DSM in Fig. 10(c) represents a specific information layer, indicating buildings' height over their specific footprint, whereas those obtained by tomographic estimators depict a whole 3-D urban scene, containing buildings, green spaces, and other 
kinds of environments. Over built-up areas, DSMs derived from VV data by using MUSIC or WSF techniques show numerous artifacts, as depicted in Fig. 10(d)-(f). No major differences may be observed between results obtained from the mono-dimensional MUSIC approach and the multidimensional WSF methods, despite their much higher complexity. DSMs obtained using fully polarimetric data sets and WSF techniques shown in Fig. 10(d) and (i) illustrate a clear enhancement with respect to their SP counterparts, due to the improved discrimination of closely spaced scatterers provided by polarimetric diversity. Nevertheless, a global underestimation of elevation, indicated by magenta contours, may be perceived on the P-NSF results, over orientated buildings as well as over some tall buildings. This is certainly due to the limitation of the P-NSF estimator in the presence of coherent scatterers. One may note that the P-MUSIC derived DSM does not demonstrate a clear improvement with respect to its SP version, whose quality was comparable to WSF products. This might be due to a worse use of the additional dimensions provided by polarimetric diversity, and shall be connected to the 1-D nature of this algorithm. The DSM derived by the P-SSF technique displayed in Fig. 10(i) provides an overall characterization of buildings which matches well with the LiDAR map of Fig. 10(c) and correspond to the highest level of quality observed among the methods tested in this study. Fig. 11 presents a detailed view of the 3-D reconstruction obtained by the P-SSF technique over some regions of interest, indicated in Fig. 11(a). Over Zone 1, buildings reconstructed by the P-SSF technique [Fig. 11(c)] are very close in appearance to the ones derived from LiDAR data [Fig. 11(b)]. Over Zone 2, the interval between horizontally and vertically aligned edifices cannot be discriminated in Fig. 11(e), due to the loss of horizontal resolution induced by spatial filtering employed during the tomographic processing. The shapes reconstructed between the buildings over Zone 3 in Fig. 11(g), are due to a bias generated by volumetric vegetation.

\section{FR Polarimetric Tomographic FocUsing}

\section{A. FR Polarimetric Tomographic Signal Model}

Over distributed environments, whose radar response is affected by the speckle phenomenon, polarimetric information is very unlikely to follow a deterministic behavior, as assumed in (5). In order to account for polarimetric variations over the different realizations of the measured vector, this model may then be modified as follows:

$$
\begin{aligned}
\mathbf{y}_{\mathrm{P}-\mathrm{FR}}(l) & =\sum_{i=1}^{n_{s}} s_{i}(l) \mathbf{a}\left(z_{i}, \mathbf{k}_{i}(l)\right)+\mathbf{n}(l) \\
& =\mathbf{A}(\mathbf{z}, \mathbf{K}(l)) \mathbf{s}(l)+\mathbf{n}(l)
\end{aligned}
$$

where the subscript FR stands for Full-Rank and the polarimetrically stochastic PolTomoSAR steering vector is given by $\mathbf{a}\left(z_{i}, \mathbf{k}_{i}(l)\right)=\mathbf{k}_{i}(l) \otimes \mathbf{a}\left(z_{i}\right)$. The covariance matrix of the received PolTomoSAR data may then be written as

$$
\mathbf{R}_{P}=\sum_{i=1}^{n_{s}} \mathbf{T}_{i} \otimes \mathbf{a}\left(z_{i}\right) \mathbf{a}^{H}\left(z_{i}\right)+\sigma_{n}^{2} \mathbf{I}_{(3 M \times 3 M)}
$$

where $\mathbf{T}_{i}$ represents the arbitrary rank covariance matrix of the polarimetric response of the $i^{\text {th }}$ scatterer.

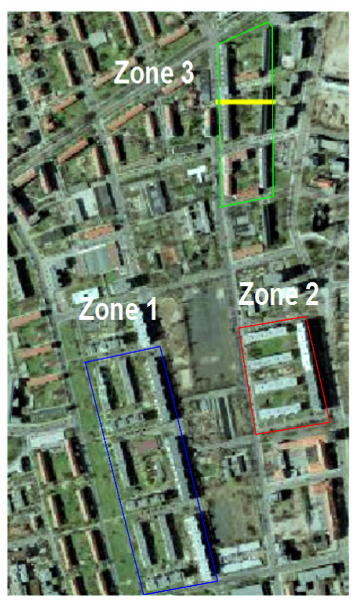

(a)

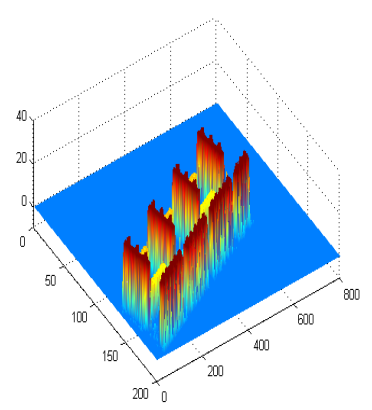

(b)

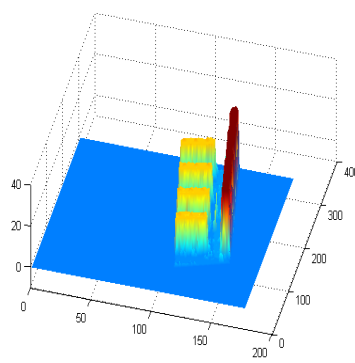

(d)

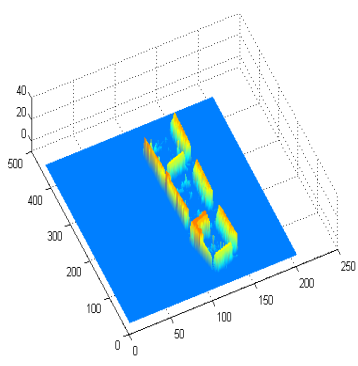

(f)

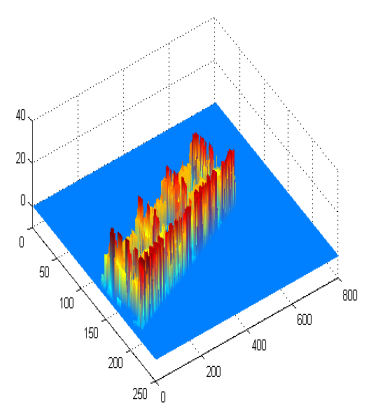

(c)

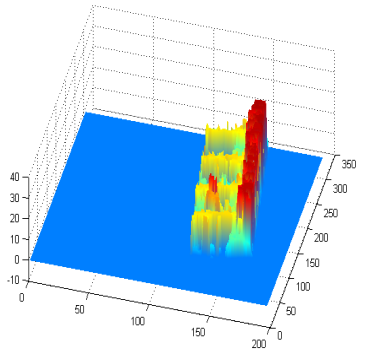

(e)

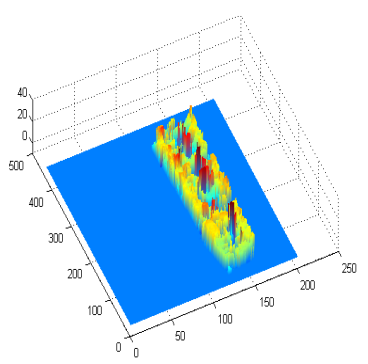

(g)
Fig. 11. Comparison of 3-D building reconstruction by P-SSF estimator with LiDAR data. (a) Google image (c). (b) Zone 1-LiDAR. (c) Zone 1-P-SSF. (d) Zone 2-LiDAR. (e) Zone 2-P-SSF. (f) Zone 3-LiDAR. (g) Zone 3-P-SSF.

\section{B. FR Polarimetric Estimators}

The objective of FR polarimetric tomography is to estimate the height $z_{i}$, reflectivity $\sigma_{i}$, and polarimetric covariance

\section{ACCEPTED MANUSCRIPT}


matrix $\mathbf{T}_{i}$ of each of the $n_{s}$ observed scatterer. Linear monodimensional FR estimators, i.e., FR-BF or FR-CP, may be defined by extending polarimetric solutions to the case of FR polarimetric quantities. Source parameters are estimated as the coordinates of the $n_{s}$ largest local maxima of a FR polarimetric objective function $P(z, \mathbf{T})$

$$
\widehat{\mathbf{z}}, \widehat{\mathbf{T}}=\arg \max _{z, \mathbf{T}, l o c} P_{\mathrm{FR}}(z, \mathbf{T}) .
$$

This estimation requires then to evaluate, for each elevation $z$, the maximal value reached by the objective function when $\mathbf{T}$ varies over the space of positive semidefinite hermitian matrices. This high-dimensional search problem may be simplified by considering unitary-rank polarimetric objectives, recalled hereafter

$$
\begin{aligned}
P_{\mathrm{P}-\mathrm{BF}}(z, \mathbf{k}) & =\frac{\mathbf{a}^{H}(z, \mathbf{k}) \widehat{\mathbf{R}}_{P} \mathbf{a}(z, \mathbf{k})}{\left(N_{p} M\right)^{2}} \\
P_{\mathrm{P}-\mathrm{CP}}(z, \mathbf{k}) & =\frac{1}{\mathbf{a}^{H}(z, \mathbf{k}) \widehat{\mathbf{R}}_{P}^{-1} \mathbf{a}(z, \mathbf{k})}
\end{aligned}
$$

together with the eigenstructure of a polarimetric covariance matrix

$$
\mathbf{T}=\mathbf{U} \boldsymbol{\Lambda} \mathbf{U}^{H}=\sum_{j=1}^{N_{P}} \lambda_{j} \mathbf{u}_{j} \mathbf{u}_{j}^{H}
$$

The objective functions in (26) correspond to estimates of the scene reflectivity for a given elevation and unitary-rank polarimetric scattering mechanism, and may be related to the polarimetric covariance matrix as follows:

$$
P_{P}(z, \mathbf{k})=\mathbf{k}^{H} \mathbf{T}(z) \mathbf{k} .
$$

This relationship may then be used to estimate $\mathbf{T}(z)$ through the determination of its eigenparameters. Optimal values of $\mathbf{k}$ provide estimates of the eigenvectors of the polarimetric covariance matrix

$$
\begin{aligned}
& \widehat{\mathbf{u}}_{1}=\arg \max _{\mathbf{k}_{1}} P_{P}\left(z, \mathbf{k}_{1}\right) \\
& \widehat{\mathbf{u}}_{2}=\arg \max _{\mathbf{k}_{2}, \mathbf{k}_{2}^{H} \widehat{\mathbf{u}}_{1}=0} P_{P}\left(z, \mathbf{k}_{2}\right)
\end{aligned}
$$

where $\mathbf{k}_{i}^{H} \mathbf{k}_{i}=1$. The eigenvalues are then determined as the reflectivity values corresponding to the different target vectors that are eigenvectors of $\mathbf{T}(z)$

$$
\hat{\lambda}_{j}=P_{P}\left(z, \widehat{\mathbf{u}}_{j}\right) \quad j=1, \ldots, N_{P} .
$$

Inserting the expression of $\mathbf{a}(z, \mathbf{k})$ in (4) into (26), one gets the expression of the polarimetric BF objective function

$$
P_{\mathrm{P}-\mathrm{BF}}(z, \mathbf{k})=\frac{\mathbf{k}^{H} \mathbf{B}_{\mathbf{a}}^{H}(z) \widehat{\mathbf{R}}_{P} \mathbf{B}_{\mathbf{a}}(z) \mathbf{k}}{\left(N_{p} M\right)^{2}} .
$$

Comparing this expression with (28) permits to conclude that the $\left(N_{P} \times N_{P}\right)$ matrix $\mathbf{B}_{\mathbf{a}}^{H}(z) \widehat{\mathbf{R}}_{P} \mathbf{B}_{\mathbf{a}}(z)$ is actually the FR-BF estimate of $\mathbf{T}(z)$. In order to account for the whole Full Rank polarimetric information, the FR-BF objective is defined as

$$
P_{\mathrm{FR}-\mathrm{BF}}(z)=\sum_{j=1}^{N_{P}} P_{\mathrm{P}-\mathrm{BF}}\left(z, \mathbf{u}_{j}\right)
$$

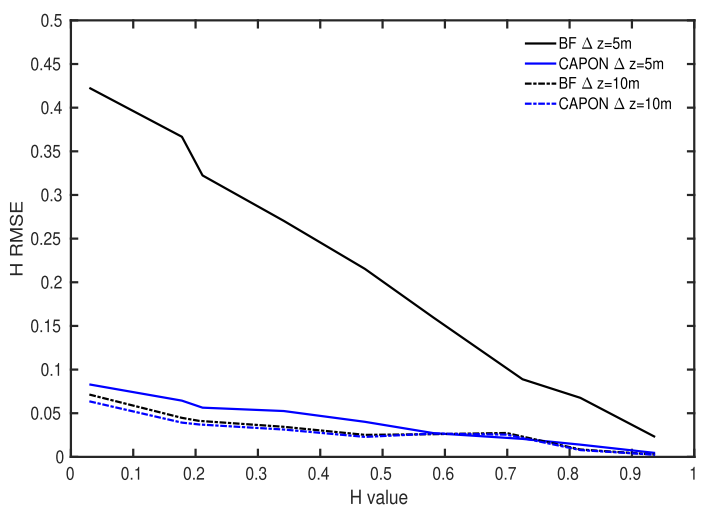

Fig. 12. Entropy RMSE obtained by FR polarimetric estimators $(M=3)$.

that is, the total polarimetric intensity estimated at elevation $z$ over $N_{P}$ orthogonal polarimetric directions, and hence, independent of the considered polarization basis. The FR-BF approach may then be summarized as

$$
\begin{aligned}
\widehat{\mathbf{z}} & =\arg \max _{z, l o c} P_{\mathrm{FR}-\mathrm{BF}}(z)=\operatorname{tr}\left(\mathbf{M}_{\mathrm{BF}}(z)\right) \\
\widehat{\mathbf{T}}_{\mathrm{BF}}(\hat{z}) & =\mathbf{M}_{\mathrm{BF}}(\mathbf{z})
\end{aligned}
$$

with

$$
\mathbf{M}_{\mathrm{BF}}(\mathbf{z})=\frac{\mathbf{B}_{\mathbf{a}}^{H}(z) \widehat{\mathbf{R}}_{P} \mathbf{B}_{\mathbf{a}}(z)}{\left(N_{p} M\right)^{2}} .
$$

Similarly, one may express the FR-CP objective function as

$$
P_{\mathrm{P}-\mathrm{CP}}(z, \mathbf{k})=\frac{1}{\mathbf{k}^{H} \mathbf{B}_{\mathbf{a}}^{H}(z) \widehat{\mathbf{R}}_{P}^{-1} \mathbf{B}_{\mathbf{a}}(z) \mathbf{k}} .
$$

Again, comparing (34) with (28), and defining the FR objective as the total polarimetric reflectivity estimated at a given height, one gets the following estimation procedure:

$$
\begin{aligned}
& \widehat{\mathbf{z}}=\arg \max _{z, l o c} P_{\mathrm{FR}-\mathrm{CP}}(z)=\operatorname{tr}\left(\mathbf{M}_{\mathrm{CP}}^{-1}(z)\right) \\
& \widehat{\mathbf{T}}_{\mathrm{CP}}(\hat{z})=\mathbf{M}_{\mathrm{CP}}^{-1}(\mathbf{z})
\end{aligned}
$$

with

$$
\mathbf{M}_{\mathrm{CP}}(\mathbf{z})=\mathbf{B}_{\mathbf{a}}^{H}(z) \widehat{\mathbf{R}}_{P}^{-1} \mathbf{B}_{\mathbf{a}}(z) .
$$

\section{Simulations}

As seen previously, nonparametric tomographic estimators have a strongly limited vertical resolution, and the estimation of FR polarimetric quantities do not modify this kind of properties. This section concentrates on their performance concerning the estimation of polarimetric parameters, such as the entropy $H$. Results obtained using the FR-Beamforming and FR-Capon estimators are summarized in Fig. 12. The accuracy of entropy estimates is evaluated for two scatterers, separated by $\Delta z=10 \mathrm{~m}$ and $\Delta z=5 \mathrm{~m}$, and for a minimal configuration with $M=3$. The performance of the FR-BF technique is strongly conditioned by the height difference $\Delta z$, whereas the FR-Capon estimator is less sensitive, due to its better resolution, and reaches lower entropy RMSE values.

\section{ACCEPTED MANUSCRIPT}


It is interesting to see that the performance of FR polarimetric estimators improves for increasing values of $H$.

Due to the lack of resolution, the estimation of 3-D polarimetric information using the Beamforming technique generally leads to poor results, whereas the quality of tomographic focusing using the FR-Capon approach has been appreciated in [21]. In order to further characterize the scattering features of urban areas, the FR-Capon technique can be hence considered as the best choice so far, since FR solutions of parametric estimators have not been developed yet. Reflectivity estimates performed using the Capon technique are known to be altered by a bias [50]. In the case of the FR-CP method, this bias affects the eigenvalues of the reconstructed polarimetric covariance matrix $\widehat{\mathbf{T}}_{\mathrm{CP}}(\hat{z})$, and may perturbate the estimated polarimetric information. As one may observe in Fig. 12, the influence of this bias on FR-CP estimates remains negligible with respect to the preponderant role played by spatial resolution. Indeed the polarimetric entropy RMSE values obtained with FR-CP are smaller than those obtained with FR-BF, which are not intrinsically biased, but suffer from a lack of spatial resolution.

\section{Application}

As shown in the previous simulations, the performance of unitary-rank polarimetric estimators may be influenced by the entropy value of the analyzed polarimetric responses. Thus, it is important to demonstrate how 3-D entropy behaves over built-up areas. The FR-Capon technique is applied over the whole test zone to estimate $H(z)$, the polarimetric entropy of each 3-D resolution cell. Fig. 13(c) and (d) illustrates a comparison between the 2-D PolSAR entropy and the estimated entropy at the ground elevation $z_{g}$, i.e., $H\left(\hat{z}_{g}\right)$ with $\hat{z}_{g}$ obtained by the P-SSF technique. It may be observed that the entropy estimated at $\hat{z}_{g}$ is obviously lower than the value of the 2-D PolSAR entropy over built-up areas, with, in general, $H\left(\hat{z}_{g}\right)<0.7$, due to the separation of scattering contributions in elevation. However, over some vegetated areas or low-intensity open areas, $H\left(\hat{z}_{g}\right)$ is close to the PolSAR entropy, with $H\left(\hat{z}_{g}\right)>0.85$, either due to the saturation of scattering patterns within a 3-D resolution cell or due to the low SNR. Three profiles are selected in the range direction as indicated in Fig. 13(a) and (b). The first one goes through a set of buildings that are orthogonal to the range axis, whereas the buildings located along the other two profiles show a nonnull orientation with respect to the azimuth direction. The entropy $H$ is obtained at the scatterers' elevations estimated by the P-SSF technique, that may be compared with LiDAR data. The resulting tomograms in Fig. 13(e)-(g) indicate that the entropy maintains a low value over bare soil and wallground interaction due to the presence of a dominant scattering mechanism within that resolution cell. However, it reaches an intermediate value over building roofs, due to a mixture of scattering features, and a high value over vegetated areas located between the buildings caused by volume scattering. Special attention may be paid to Profile 3, over which the entropy derived by 2-D PolSAR technique seems to be

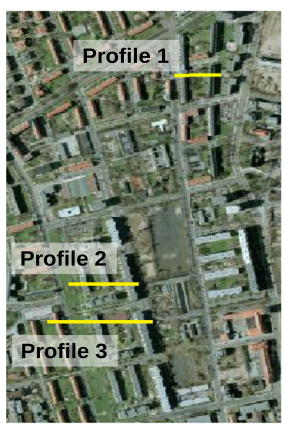

(a)

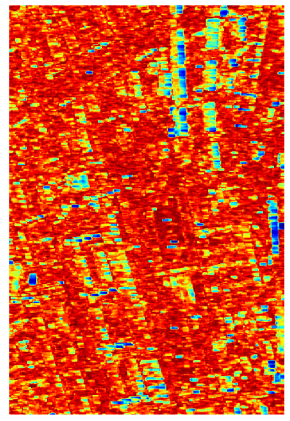

(c)

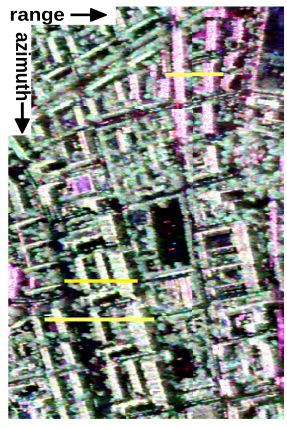

(b)

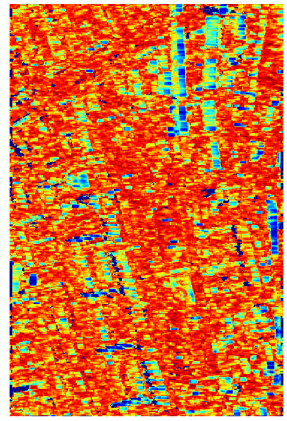

(d)

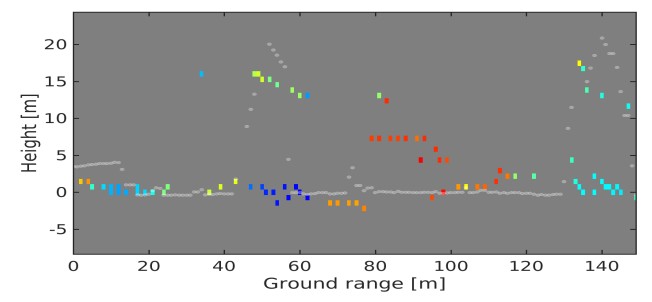

(e)

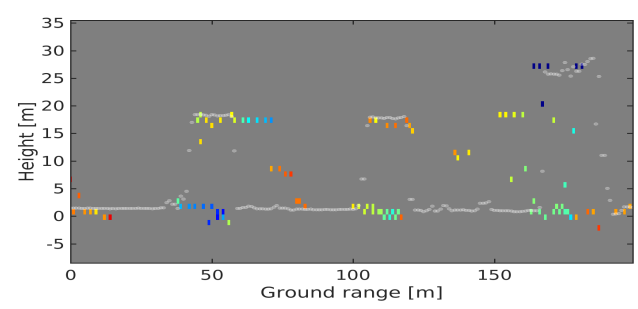

(f)

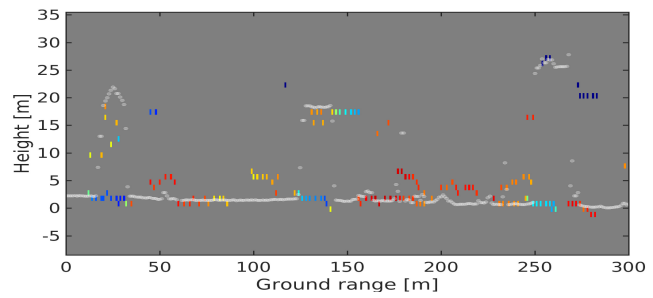

(g)

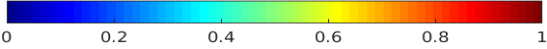

Fig. 13. P-SSF tomograms in ground range with entropy values estimated by the FR-Capon estimator. LiDAR-derived building heights are indicated by a white dotted line. (a) Optical image in 2000. (b) Pauli-based image. (c) 2-D entropy. (d) Entropy at ground level: $H\left(\hat{z}_{g}\right)$. (e) Entropy $H\left(\hat{z}_{i}\right)$ over Profile 1. (f) Entropy $H\left(\hat{z}_{i}\right)$ over Profile 2. (g) Entropy $H\left(\hat{z}_{i}\right)$ over Profile 3.

\section{ACCEPTED MANUSCRIPT}


saturated for oriented buildings [Fig. 13(c)]. However, using the FR-Capon technique, it is possible to discriminate these oriented buildings from the vegetated areas in Fig. 13(f) as they show lower 3-D entropy values.

\section{CONCLUSION}

SAR tomographic focusing for 3-D imaging using a minimal configuration, i.e., three images only, and intermediate-resolution SAR data, is a challenging application, which may reveal useful for the characterization of numerous complex urban environments, over which only a few images were acquired in an adequate configuration. This article shows that some performing processing techniques may be designed, that make use of polarimetric diversity, and adapt well to such extreme configurations, both theoretically and using actually measured signals. Numerical simulations considering different numbers of acquisitions, or values of source correlation and SNR, indicate that conventional single- or multipolarization tomographic focusing techniques are differently affected by these factors. In virtue of the relationship between vertical resolution, ambiguity, and number of images, nonparametric techniques, known for their robustness to the presence of noise or nonnominal signal patterns, reveal badly adapted to this kind of applications due to their limited resolution of analysis. Among HR estimation techniques, multidimensional approaches such as ML and WSF perform better than mono-dimensional ones, such as MUSIC, in the presence of coherent or closely spaced scatterers. Compared with the SML method, the DML undergoes a significant degradation of performance over coherent scatterers, especially in the low SNR case, whereas WSF techniques maintain an asymptotic equivalent performance. The SSF estimator outperforms other HR parametric estimators, whatever the source correlation or SNR values, due both to its model-adaptivity, and to the fact that it is not based on the orthogonality of the received signals with a noise subspace, whose definition becomes ambiguous in the presence of correlated sources. In a minimal tomographic configuration, the use of polarization diversity plays an important role in the discrimination of closely spaced scatterers. The amount of improvement that may be expected from polarimetric processing depends on the entropy, or polarimetric randomness, of the observed source responses, and may reach extremely high levels when measuring fully polarized signals, $H \approx 0$, whereas unpolarized features, $H \approx 1$, do not lead to better discrimination with respect to single-polarization measurements. From these observations, one may naturally conclude that the P-SSF estimator is, among the investigated solutions, the most attractive solution, as it combines the use of polarimetric diversity with excellent performance in terms of scatterer discrimination in a minimal configuration and at low SNR case. A new iterative optimization technique, P-AP, based on alternating projections over polarimetric and spatial subspaces is derived, which permits to solve the P-SSF and the $\mathrm{P}-\mathrm{DML}$ criteria in a similar way. The P-SSF is then shown to outperform all the other studied polarimetric HR techniques and its application to real data lead to satisfying reconstruction results, using three polarimetric SAR images only. FR nonparametric tomographic estimators are proposed, which permit further characterize reconstructed urban areas. Unlike classical unitary rank approaches, they are able to estimate at 3-D complex polarimetric scattering mechanisms with an intermediate vertical resolution. These simple techniques are used to discriminate entangled vegetated and built-up areas and allow to export any polarimetric signal processing techniques developed for 2-D images in the 3-D world.

\section{ACKNOWLEDGMENT}

The authors would like to thank the ESAR Team of the DLR for their efforts in data acquisition and processing, and Dr. A. Reigber for his kind support.

\section{REFERENCES}

[1] G. Fornaro and F. Serafino, "Imaging of single and double scatterers in urban areas via SAR tomography," IEEE Trans. Geosci. Remote Sens., vol. 44, no. 12, pp. 3497-3505, Dec. 2006.

[2] A. Budillon, A. Evangelista, and G. Schirinzi, "Three-dimensional SAR focusing from multipass signals using compressive sampling," IEEE Trans. Geosci. Remote Sens., vol. 49, no. 1, pp. 488-499, Jan. 2011.

[3] X. X. Zhu and R. Bamler, "Tomographic SAR inversion by $L_{1}$-norm regularization-The compressive sensing approach," IEEE Trans. Geosci. Remote Sens., vol. 48, no. 10, pp. 3839-3846, Oct. 2010.

[4] S. Sauer, L. Ferro-Famil, A. Reigber, and E. Pottier, "Three-dimensional imaging and scattering mechanism estimation over urban scenes using dual-baseline polarimetric InSAR observations at L-band," IEEE Trans. Geosc. Remote Sens., pp. 1-14, Jun. 2011.

[5] S. Guillaso, O. D'Hondt, and O. Hellwich, "Urban scene reconstruction from a reduced number of tomographic SAR data," in Proc. IEEE Int. Geosci. Remote Sens. Symp. (IGARSS), Jul. 2014, pp. 3164-3167.

[6] Y. Huang and L. Ferro-Famil, "3-D characterization of buildings in a dense urban environment using L-band pol-InSAR data with irregular baselines," in Proc. IEEE Int. Geosci. Remote Sens. Symp., Jul. 2009, p. 29.

[7] O. D'Hondt, C. Lopez-Martinez, S. Guillaso, and O. Hellwich, "Nonlocal filtering applied to $3-\mathrm{D}$ reconstruction of tomographic SAR data," IEEE Trans. Geosci. Remote Sens., vol. 56, no. 1, pp. 272-285, Jan. 2018.

[8] F. Gini and F. Lombardini, "Multibaseline cross-track SAR interferometry: A signal processing perspective," IEEE Aerosp. Electron. Syst. Mag., vol. 20, no. 8, pp. 71-93, Aug. 2005.

[9] A. Reigber and A. Moreira, "First demonstration of airborne SAR tomography using multibaseline L-band data," IEEE Trans. Geosci. Remote Sens., vol. 38, no. 5, pp. 2142-2152, Sep. 2000.

[10] F. Gini, F. Lombardini, and M. Montanari, "Layover solution in multibaseline SAR interferometry," IEEE Trans. Aerosp. Electron. Syst., vol. 38, no. 4, pp. 1344-1356, Oct. 2002.

[11] C.-A. Deledalle, L. Denis, F. Tupin, A. Reigber, and M. Jager, "NL-SAR: A unified nonlocal framework for resolution-preserving (Pol)(In)SAR denoising," IEEE Trans. Geosci. Remote Sens., vol. 53, no. 4, pp. 2021-2038, Apr. 2015.

[12] O. D'Hondt, S. Guillaso, and O. Hellwich, "Iterative bilateral filtering of polarimetric SAR data," IEEE J. Sel. Topics Appl. Earth Observ. Remote Sens., vol. 6, no. 3, pp. 1628-1639, Jun. 2013.

[13] Y. Shi, R. Bamler, Y. Wang, and X. X. Zhu, "Sar tomography at the limit: Building height reconstruction using only 3-5 Tandem-x bistatic interferograms," IEEE Trans. Geosci. Remote Sens., vol. 58, no. 11, pp. 8026-8037, Nov. 2020.

[14] A. Ferretti, C. Prati, and F. Rocca, "Permanent scatterers in SAR interferometry," IEEE Trans. Geosci. Remote Sens., vol. 39, no. 1, pp. 8-20, Jan. 2001.

[15] S. Sauer, L. Ferro-Famil, A. Reigber, and E. Pottier, "Polarimetric dualbaseline InSAR building height estimation at L-band," IEEE Geosci. Remote Sens. Lett., vol. 6, no. 3, pp. 408-412, Jul. 2009.

[16] S. Guillaso, L. Ferro-Famil, A. Reigber, and E. Pottier, "Building characterization using L-Band polarimetric interferometric SAR data," IEEE Geosci. Remote Sens. Lett., vol. 2, no. 3, pp. 347-351, Jul. 2005.

[17] Y. Huang and L. Ferro-Famil, "Building height estimation using multibaseline 1-band SAR data and polarimetric weighted subspace fitting methods," in Proc. PolInSAR Conf., Jan. 2009. 
[18] Y. Huang, L. Ferro-Famil, and A. Reigber, "Under-foliage object imaging using SAR tomography and polarimetric spectral estimators," IEEE Trans. Geosci. Remote Sens., vol. 50, no. 6, pp. 2213-2225, Jun. 2012.

[19] A. Swindlehurst and M. Viberg, "Subspace fitting with diversely polarized antenna arrays," IEEE Trans. Antennas Propag., vol. 41, no. 12, pp. 1687-1694, Dec. 1993.

[20] L. Ferro-Famil and E. Pottier, "Urban area remote sensing from Lband polsar data using time-frequency techniques," in Proc. IEEE Urban Remote Sens. Joint Event, Apr. 2007, pp. 5045-5048.

[21] L. Ferro-Famil, Y. Huang, and A. Reigber, "High-resolution SAR tomography using full rank polarimetric spectral estimators," in Proc. IEEE IGARSS, Jul. 2012, pp. 5194-5197.

[22] Y. Huang, L. Ferro-Famil, and A. Reigber, "Under-foliage target detection using multi-baseline L-band PolInSAR data," in Proc. IEEE Int. Geosci. Remote Sens. Symp. IGARSS, Jul. 2013, pp. 2353-2356.

[23] Y. Huang, J. Levy-Vehel, L. Ferro-Famil, and A. Reigber, "Threedimensional imaging of objects concealed below a forest canopy using SAR tomography at L-band and wavelet-based sparse estimation," IEEE Geosci. Remote Sens. Lett., vol. 14, no. 9, pp. 1454-1458, Sep. 2017.

[24] H. Aghababaee, G. Ferraioli, L. Ferro-Famil, G. Schirinzi, and Y. Huang, "Sparsity based full rank polarimetric reconstruction of coherence matrix t," Remote Sens., vol. 11, no. 11, p. 1288, May 2019.

[25] P. Stoica and A. Nehorai, "Performance study of conditional and unconditional direction-of-arrival estimation," IEEE Trans. Acoust., Speech, Signal Process., vol. 38, no. 10, pp. 1783-1795, Oct. 1990.

[26] L. Ferro-Famil, A. Reigber, and E. Pottier, "Nonstationary natural media analysis from polarimetric sar data using a two-dimensional timeFrequency decomposition approach," Can. J. Remote Sens., vol. 31, no. 1, pp. 9-21, 2005.

[27] J. S. Lee and E. Pottier, Polarimetric Radar Imaging: From Basics to Applications. New York, NY, USA: CRC Press, Feb. 2009.

[28] M. Nannini, R. Scheiber, and A. Moreira, "Estimation of the minimum number of tracks for SAR tomography," IEEE Trans. Geosci. Remote Sens., vol. 47, no. 2, pp. 531-543, Feb. 2009.

[29] P. Stoica and A. Nehorai, "Music, maximum likelihood, and cramer-rao bound," IEEE Trans. Acoust. Speech Signal Process., vol. 37, no. 5, pp. 702-741, May 1989.

[30] P. Stoica and A. Nehorai, "MUSIC, maximum likelihood, and cramer-rao bound: Further results and comparisons," IEEE Trans. Acoust., Speech, Signal Process., vol. 38, no. 12, pp. 2140-2150, Dec. 1990.

[31] P. Stoica, B. Ottersten, M. Viberg, and R. L. Moses, "Maximum likelihood array processing for stochastic coherent sources," IEEE Trans. Signal Process., vol. 44, no. 1, pp. 96-105, Jan. 1996.

[32] J. Capon, "High-resolution frequency-wavenumber spectrum analysis," Proc. IEEE, vol. 57, no. 8, pp. 1408-1418, Aug. 1969.

[33] A. Jakobsson, F. Gini, and F. Lombardini, "Layover solution in multibaseline INSAR using robust beamforming," in Proc. 3rd IEEE Int. Symp. Signal Process. Inf. Technol., Dec. 2003, pp. 328-331.

[34] F. Lombardini, J. Ender, L. Rossing, M. Galletto, and L. Verrazzani, "Experiments of interferometric layover solution with the three-antenna airborne AER-II SAR system," in Proc. IEEE Int. IEEE Int. IEEE Int. Geosci. Remote Sens. Sy, Sep. 2004, pp. 3341-3344.

[35] R. Schmidt, "Multiple emitter location and signal parameter estimation," IEEE Trans. Antennas Propag., vol. 34, no. 3, pp. 276-280, Mar. 1986.

[36] P. Stoica and R. Moses, Spectral Analysis of Signals. Upper Saddle River, NJ, USA: Prentice-Hall, 2005.

[37] F. Lombardini, M. Montanari, and F. Gini, "Reflectivity estimation for multibaseline interferometric radar imaging of layover extended sources," IEEE Trans. Signal Process., vol. 51, no. 6, pp. 1508-1519, Jun. 2003.

[38] B. Ottersten, M. Viberg, P. Stoica, and A. Nehorai, "Exact and large sample $\mathrm{ml}$ techniques for parameter estimation and detection in array processing," Radar Array Process., Berlin, Germany: Springer, 1993, pp. $99-151$.

[39] P. Stoica and K. C. Sharman, "Maximum likelihood methods for direction-of-arrival estimation," IEEE Trans. Acoust., Speech, Signal Process., vol. 38, no. 7, pp. 1132-1143, Jul. 1990.

[40] M. Schmitt and U. Stilla, "Maximum-likelihood-based approach for single-pass synthetic aperture radar tomography over urban areas," IET Radar, Sonar Navigat., vol. 8, no. 9, pp. 1145-1153, Dec. 2014.

[41] M. Viberg and B. Ottersten, "Sensor array processing based on subspace fitting," IEEE Trans. Signal Process., vol. 39, no. 5, pp. 1110-1121, May 1991.

[42] E. Ferrara and T. Parks, "Direction finding with an array of antennas having diverse polarizations," IEEE Trans. Antennas Propag., vol. AP-31, no. 2, pp. 231-236, Mar. 1983
[43] A. J. Weiss and B. Friedlander, "Direction finding for diversely polarized signals using polynomial rooting," IEEE Trans. Signal Process., vol. 41, no. 5, pp. 1893-1905, May 1993.

[44] M. Wax, "Detection and localization of multiple sources via the stochastic signals model," IEEE Trans. Signal Process., vol. 39, no. 11, pp. 2450-2456, Nov. 1991.

[45] S. R. Cloude and E. Pottier, "A review of target decomposition theorems in radar polarimetry," IEEE Trans. Geosci. Remote Sens., vol. 34, no. 2, pp. 498-518, Mar. 1996.

[46] C. Hu, L. Ferro-Famil, and G. Kuang, "Ship discrimination using polarimetric SAR data and coherent time-frequency analysis," Remote Sens., vol. 5, no. 12, pp. 6899-6920, Dec. 2013.

[47] A. Paulraj, B. Ottersten, R. Roy, A. Swindlehurst, G. Xu, and T. Kailath, "Subspace methods for directions-of-arrival estimation," in Signal Processing and its Applications (Handbook of Statistics), vol. 10. Amsterdam, The Netherlands: Elsevier, 1993, pp. 693-739.

[48] L. Ferro-Famil, Y. Huang, and E. Pottier, Principles and Applications of Polarimetric SAR Tomography for the Characterization of Complex Environments. Berlin, Germany: Springer, 2015, pp. 1-13.

[49] I. Ziskind and M. Wax, "Maximum likelihood localization of multiple sources by alternating projection," IEEE Trans. Acoust., Speech, Signal Process., vol. 36, no. 10, pp. 1553-1560, Oct. 1988.

[50] P. Stoica, A. Jakobsson, and J. Li, "Matched-filter bank interpretation of some spectral estimators," Signal Process., vol. 66, no. 1, pp. 45-59, Apr. 1998

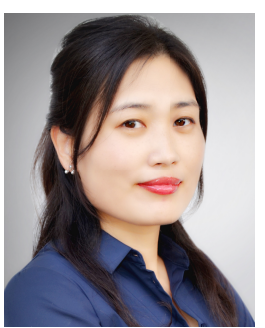

Yue Huang (Member, IEEE) received the M.Sc. degree in electronics, electrical engineering, and automation and systems from Paul Sabatier University, Toulouse, France, in 2007, and the Ph.D. degree in signal processing and telecommunications from the University of Rennes 1, Rennes, France, in 2011.

She is with the Institute of Electronics and Telecommunications of Rennes, Rennes. From 2011 to 2015, she was a Senior Scientist with Intermap Technologies Corporation, Calgary, $\mathrm{AB}$, Canada. She has been involved in many Research and Development Projects of synthetic-aperture radar (SAR) imaging and its applications. Her research interests include polarimetric SAR interferometry techniques, SAR interferometry and differential interferometry, physical parameter retrieval, 3-D environment reconstruction, target detection, SAR signal processing, SAR tomography, multivariate spectral estimation methods, and blind source separation.

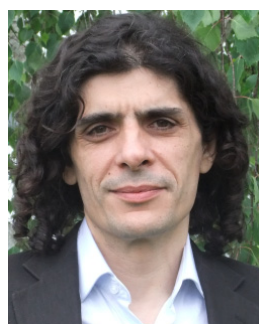

Laurent Ferro-Famil (Member, IEEE) received the Laurea degree in electronics systems and computer engineering and the M.S. and Ph.D. degrees in electronics from the University of Nantes, Nantes, France, in 1996, 1996, and 2000, respectively.

In 2001, he became an Associate Professor with the University of Rennes 1, Toulouse, France, where he has been a Full Professor, since 2011. He is the Head of the Waves and Signals Department, IETR Laboratory, Rennes, and also with the Radar Group of CESBIO Laboratory, Toulouse. His activities in education are concerned with analog electronics, digital communications, microwave theory, signal processing, and polarimetric Synthetic Aperture Radar (SAR) remote sensing. His research interests include radar signal processing for remote sensing application, including radar polarimetry theory, electromagnetic imaging and natural media remote sensing using radar multibaseline PolInSAR data, with application to classification, electromagnetic scattering modeling, physical parameter retrieval, and 3-D reconstruction of environments using tomography. 